\title{
A model of electroweakly interacting non-abelian vector dark matter
}

\author{
Tomohiro Abe, ${ }^{a, b}$ Motoko Fujiwara, ${ }^{c}$ Junji Hisano ${ }^{b, c, d}$ and Kohei Matsushita ${ }^{c}$ \\ ${ }^{a}$ Institute for Advanced Research, Nagoya University, \\ Furo-cho Chikusa-ku, Nagoya, Aichi, 464-8602 Japan \\ ${ }^{b}$ Kobayashi-Maskawa Institute for the Origin of Particles and the Universe, \\ Nagoya University, Furo-cho Chikusa-ku, Nagoya, 464-8602 Japan \\ ${ }^{c}$ Department of Physics, Nagoya University, \\ Furo-cho Chikusa-ku, Nagoya, 464-8602 Japan \\ ${ }^{d}$ Kavli IPMU (WPI), UTIAS, University of Tokyo, \\ Kashiwa, 277-8584, Japan \\ E-mail: abetomo@kmi.nagoya-u.ac.jp, motoko@eken.phys.nagoya-u.ac.jp, \\ hisano@eken.phys.nagoya-u.ac.jp, kohei@eken.phys.nagoya-u.ac.jp
}

ABSTRACT: We propose an electroweakly interacting spin-1 dark matter (DM) model. The electroweak gauge symmetry, $\mathrm{SU}(2)_{L} \times \mathrm{U}(1)_{Y}$, is extended into $\mathrm{SU}(2)_{0} \times \mathrm{SU}(2)_{1}$ $\times \mathrm{SU}(2)_{2} \times \mathrm{U}(1)_{Y}$. A discrete symmetry exchanging $\mathrm{SU}(2)_{0}$ and $\mathrm{SU}(2)_{2}$ is imposed. This discrete symmetry stabilizes the DM candidate. The spin-1 DM particle $\left(V^{0}\right)$ and its $\mathrm{SU}(2)_{L}$ partners $\left(V^{ \pm}\right)$interact with the Standard Model (SM) electroweak gauge bosons without any suppression factors. Consequently, pairs of DM particles efficiently annihilate into the SM particles in the early universe, and the measured value of the DM energy density is easily realized by the thermal freeze-out mechanism. The model also predicts a heavy vector triplet $\left(W^{\prime \pm}\right.$ and $Z^{\prime}$ ) in the visible sector. They contribute to the DM annihilation processes. The mass ratio of $Z^{\prime}$ and $V^{0}$ determines values of various couplings, and constraints on $W^{\prime}$ and $Z^{\prime}$ restrict regions of the parameter space that are viable for DM physics. We investigate the constraints from perturbative unitarity of scalar and gauge couplings, the Higgs signal strength, $W^{\prime}$ search at the LHC, and DM direct detection experiments. It is found that the relic abundance of $V^{0}$ explains the right amount of the DM energy density for $3 \mathrm{TeV} \lesssim m_{V^{0}} \lesssim 19 \mathrm{TeV}$.

Keywords: Beyond Standard Model, Cosmology of Theories beyond the SM, Gauge Symmetry

ARXIV EPRINT: 2004.00884 


\section{Contents}

1 Introduction 1

2 Model 3

2.1 Bosonic sector 5

2.2 Gauge sector 5

2.3 Physical scalars 6

$\begin{array}{ll}2.4 \text { Model parameters } & 7\end{array}$

3 Constraints $\quad 9$

3.1 Perturbative unitarity 9

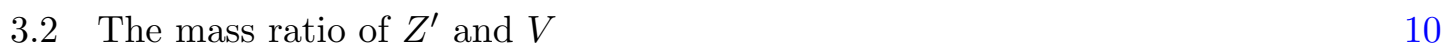

$3.3 W^{\prime}$ and $Z^{\prime}$ searches at the LHC 11

$\begin{array}{ll}3.4 & \text { Electroweak precision measurements } \\ 3.5 & 12\end{array}$

$\begin{array}{ll}3.5 & \text { Higgs signal strength } \\ \end{array}$

4 DM phenomenology 13

$\begin{array}{lll}\text { 4.1 Mass difference and its implication for collider physics } & 13\end{array}$

$\begin{array}{lll}4.2 & \text { Direct detection } & 14\end{array}$

$\begin{array}{lll}4.3 & \text { Relic abundance } & 16\end{array}$

$\begin{array}{lll}\text { 4.3.1 Very small }\left|\phi_{h}\right| \text { case } & 16\end{array}$

$\begin{array}{lll}\text { 4.3.2 } & \text { For }\left|\phi_{h}\right| \sim \mathcal{O}(0.1) & 18\end{array}$

5 Conclusions $\quad 19$

$\begin{array}{ll}\text { A Some details in the gauge sectors } & 21\end{array}$

B Would-be NG bosons $\quad 23$

$\begin{array}{ll}\text { C Fermi constant } & 24\end{array}$

\section{Introduction}

Dark matter (DM) is a longstanding issue in both particle physics and cosmology. The DM energy density is precisely measured by the Planck collaboration, $\Omega h^{2}=0.120 \pm$ 0.001 [1]. A popular scenario that explains this measured value is the thermal freeze-out scenario [2], which utilizes a pair annihilation/creation of DM particles into/from particles in the thermal bath in the early universe. This scenario requires interactions between DM and Standard Model (SM) particles. Hence, models that utilize the freeze-out mechanism are good targets of DM direct detection experiments. However, there are no significant DM 
signals at the experiments so far. The latest result by the XENON1T experiment gives a strong upper bound on the DM-nucleon scattering cross section [3]. This result implies that the DM-nucleon scattering processes mediated by the $Z$-boson and scalar mediators, such as the SM Higgs boson, must be suppressed if the mass of DM is between $\mathcal{O}(10) \mathrm{GeV}$ and $\mathcal{O}(1) \mathrm{TeV}$.

Models that predict the suppression in those processes without suppressed DMmediator coupling are proposed. Fermionic DM models with pseudo-scalar couplings are examples in spin-1/2 DM models [4-6]. The pseudo-Nambu-Goldstone (NG) boson DM models are examples of spin-0 DM models [7-10]. In these models, the coupling itself between DM and mediator particle is not suppressed, and thus DM is thermally produced in the early universe through the annihilation into the SM particles via scalar mediator exchanges.

In this paper, we propose a renormalizable model of spin-1 DM that does not require $Z$ and Higgs couplings to a DM particle to obtain the correct amount of the DM density by the freeze-out mechanism. ${ }^{1}$ We extend the electroweak gauge symmetry in the SM, $\mathrm{SU}(2)_{L} \times \mathrm{U}(1)_{Y}$, into $\mathrm{SU}(2)_{0} \times \mathrm{SU}(2)_{1} \times \mathrm{SU}(2)_{2} \times \mathrm{U}(1)_{Y}$ and impose that the model is symmetric under exchanging of $\mathrm{SU}(2)_{0}$ and $\mathrm{SU}(2)_{2}$. This symmetry predicts a stable $\mathrm{SU}(2)_{L}$ triplet vector boson, $V^{0}$ and $V^{ \pm}$. After the symmetry breaking, the charged vector boson, $V^{ \pm}$, gets slightly heavier than the neutral one, $V^{0}$, and thus $V^{0}$ is a DM candidate in our model. The vector DM in our model can directly couple to the SM weak gauge bosons and efficiently annihilate in the early universe even without the DM-Higgs coupling. The $V^{0}$ $V^{0}-Z$ coupling is automatically forbidden by the gauge symmetry. Therefore, the model easily evades the constraint from the XENON1T experiment and has a large region of viable parameter space.

There are many spin-1 DM models, but they are originated from a U(1) gauge symmetry [13-23] or an $\mathrm{SU}(2)$ gauge symmetry that is isolated from the SM electroweak sector [24-33]. Therefore, they rely on the scalar exchanges that require the mixing between the SM Higgs and new scalar particles to obtain the measured value of the DM energy density. The scalar mixing, however, is constrained from the direct detection experiments. On the other hand, our model does not require the scalar mixing for the DM energy density. This is a different feature of our model from the other spin-1 DM models. Another aspect of our model is that new spin-1 particles are predicted in the visible sector as well as the dark sector. Those new spin-1 particles in the visible sector are regarded as $W^{\prime}$ and $Z^{\prime}$. They play an important role in the DM annihilation processes. Moreover, the fermion sector of our model is as simple as in the SM. We do not need to introduce new fermions into the model to obtain the realistic mass spectra for the SM fermions. ${ }^{2}$

We organize the rest of this paper as follows. In section 2, we describe our model. Some technical details are discussed in appendices. In section 3 , we discuss constraints on the model from perturbative unitarity, the mass ratio of $Z^{\prime}$ and $V^{0}, W^{\prime}$ and $Z^{\prime}$ searches at the LHC, electroweak precision measurements, and the Higgs coupling measurements at

\footnotetext{
${ }^{1}$ Non-renormalizable models for the electroweakly interacting spin-1 DM are discussed in [11, 12].

${ }^{2}$ Non-abelian vector DM with an extended fermion sector are discussed in [26, 34-36].
} 
the LHC. After constraining the model parameters, we discuss the phenomenology of DM in section 4 . We start by discussing the mass difference between $V^{ \pm}$and $V^{0}$. As discussed later, $V^{ \pm}$is one of the targets for long-lived particle searches at the LHC. After that, we discuss the thermal relic abundance in this model. We also address the constraint from the XENON1T experiment. We show that the viable mass range of $V^{0}$ as a thermal relic is $3 \mathrm{TeV} \lesssim m_{V^{0}} \lesssim 19 \mathrm{TeV}$. Section 5 is devoted to our conclusions.

\section{Model}

The gauge symmetry is $\mathrm{SU}(3)_{c} \times \mathrm{SU}(2)_{0} \times \mathrm{SU}(2)_{1} \times \mathrm{SU}(2)_{2} \times \mathrm{U}(1)_{Y}$ in our Model. Here, $\mathrm{SU}(3)_{c}$ is for the QCD as in the same as the SM. The matter and Higgs fields are summarized in table $1 .^{3}$ In this section, we focus on the extended electroweak gauge sector, namely $\mathrm{SU}(2)_{0} \times \mathrm{SU}(2)_{1} \times \mathrm{SU}(2)_{2} \times \mathrm{U}(1)_{Y}$. We denote the gauge fields of them as $W_{0 \mu}^{a}, W_{1 \mu}^{a}$, $W_{2 \mu}^{a}$, and $B_{\mu}$, respectively, where $a=1,2,3$. Their gauge couplings are $g_{0}, g_{1}, g_{2}$, and $g^{\prime}$, respectively. The gauge transformation of two Higgs fields, $\Phi_{1}$ and $\Phi_{2}$, are given by

$$
\begin{aligned}
& \Phi_{1} \rightarrow U_{0} \Phi_{1} U_{1}^{\dagger}, \\
& \Phi_{2} \rightarrow U_{2} \Phi_{2} U_{1}^{\dagger},
\end{aligned}
$$

where $U_{j}$ 's are two-by-two unitary matrices of the $\mathrm{SU}(2)_{j}$ gauge transformation. To reduce the number of degrees of freedom, we impose

$$
\Phi_{j}=-\epsilon \Phi_{j}^{*} \epsilon, \quad \text { where } \quad \epsilon=\left(\begin{array}{cc}
0 & 1 \\
-1 & 0
\end{array}\right) .
$$

Before imposing this constraint, $\Phi_{1}$ and $\Phi_{2}$ contain four complex degrees of freedom (eight real degrees of freedom), respectively. After imposing this constraint, each field has four real degrees of freedom as shown later in eq. (2.12). This constraint has nothing to do with the dark matter stability.

We impose the following discrete symmetry.

$$
\begin{aligned}
& q_{L} \rightarrow q_{L}, \quad u_{R} \rightarrow u_{R}, \quad d_{R} \rightarrow d_{R}, \\
& \ell_{L} \rightarrow \ell_{L}, \quad e_{R} \rightarrow e_{R}, \\
& H \rightarrow H, \quad \Phi_{1} \rightarrow \Phi_{2}, \quad \Phi_{2} \rightarrow \Phi_{1}, \\
& W_{0 \mu}^{a} \rightarrow W_{2 \mu}^{a}, \quad W_{1 \mu}^{a} \rightarrow W_{1 \mu}^{a}, \quad W_{2 \mu}^{a} \rightarrow W_{0 \mu}^{a} .
\end{aligned}
$$

This discrete symmetry is equivalent to the exchange of $\mathrm{SU}(2)_{0}$ and $\mathrm{SU}(2)_{2}$. It requires $g_{0}=g_{2}$. The symmetry works as a $Z_{2}$ symmetry that is utilized in many dark matter models. Linear combinations $\left(W_{0 \mu}^{a}-W_{2 \mu}^{a}\right) / \sqrt{2}$ are odd under the symmetry. They are mass eigenstates as we will see below, and one of them is a DM candidate. On the other hand, the other linear combinations of the gauge fields are even under the symmetry. Similarly, linear combinations of $\Phi_{1}$ and $\Phi_{2}$ divide scalar fields into the odd and even sectors. All the SM particles are even under the discrete symmetry.

\footnotetext{
${ }^{3} \mathrm{~A}$ model with a similar gauge group is studied in [37] but with different matter contents and with different gauge charge assignments.
} 


\begin{tabular}{|cc|ccccc|}
\hline field & spin & $\mathrm{SU}(3)_{c}$ & $\mathrm{SU}(2)_{0}$ & $\mathrm{SU}(2)_{1}$ & $\mathrm{SU}(2)_{2}$ & $\mathrm{U}(1)_{Y}$ \\
\hline$q_{L}$ & $\frac{1}{2}$ & 3 & 1 & 2 & 1 & $\frac{1}{6}$ \\
$u_{R}$ & $\frac{1}{2}$ & 3 & 1 & 1 & 1 & $\frac{2}{3}$ \\
$d_{R}$ & $\frac{1}{2}$ & 3 & 1 & 1 & 1 & $-\frac{1}{3}$ \\
$\ell_{L}$ & $\frac{1}{2}$ & 1 & 1 & 2 & 1 & $-\frac{1}{2}$ \\
$e_{R}$ & $\frac{1}{2}$ & 1 & 1 & 1 & 1 & -1 \\
\hline$H$ & 0 & 1 & 1 & 2 & 1 & $\frac{1}{2}$ \\
$\Phi_{1}$ & 0 & 1 & 2 & 2 & 1 & 0 \\
$\Phi_{2}$ & 0 & 1 & 1 & 2 & 2 & 0 \\
\hline
\end{tabular}

Table 1. The matter and Higgs fields and their gauge charges in the model. The generation indices for the matter fields are implicit.

The discrete symmetry under exchanging $\mathrm{SU}(2)_{0}$ and $\mathrm{SU}(2)_{2}$ is inspired by the deconstruction [38, 39] of models in extra dimension on $S^{1} / Z_{2}$. Using the deconstruction approach, such models are expressed by moose diagrams [40]. The $Z_{2}$ symmetry is realized by identifying two sites. Some models with the gauge symmetry $G=\mathrm{SU}(2)_{0} \times \mathrm{SU}(2)_{1} \times$ $\cdots \times \mathrm{SU}(2)_{2 N}$ with identifying $\mathrm{SU}(2)_{j}$ and $\mathrm{SU}(2)_{2 N-j}$ are equivalent to the models in extra dimension on $S^{1} / Z_{2}$ upto $2 N$ Kaluza-Klein (KK) modes. The $\mathrm{SU}(2)$ sector in our model corresponds to the case for $N=1$. The similar approach was taken in studying a $\mathrm{U}(1)$ vector dark matter model [16].

Under this setup, we can write the Yukawa interaction terms as

$$
-y_{u} \bar{q}_{L} \tilde{H} u_{R}-y_{d} \bar{q}_{L} H d_{R}-y_{e} \bar{\ell}_{L} H e_{R}+\text { (h.c.), }
$$

where $\tilde{H}=\epsilon H^{*}$. The gauge symmetry forbids $\Phi_{1}$ and $\Phi_{2}$ to couple to the fermions, and only $H$ is the relevant Higgs field for the Yukawa interaction terms. This Yukawa sector is as simple as one in the SM, and we do not need to extend the fermion sector. This is a reason why we add two extra $\mathrm{SU}(2)$ gauge symmetries into the SM. If we added only one extra $\mathrm{SU}(2)$, there would be two possibilities. One possibility is that the extra $\mathrm{SU}(2)$ is isolated and does not mix with the $\mathrm{SU}(2)_{L}$ gauge field. In this case, the dark $\mathrm{SU}(2)$ gauge bosons do not couple to the SM weak gauge bosons, and the model is the Higgs portal type. This is not our concern. The other possibility is to mix the extra $\mathrm{SU}(2)$ gauge field with the $\mathrm{SU}(2)$ gauge field in the $\mathrm{SM}$. It is expected by the mixing that the dark $\mathrm{SU}(2)$ gauge bosons couple to the SM weak gauge bosons. In this case, however, we need an exchanging symmetry under these two $\mathrm{SU}(2)$ gauge field to stabilize the dark matter. Since the SM left-handed fermions feel $\mathrm{SU}(2)_{L}$ gauge symmetry, the symmetry exchanging the two $\mathrm{SU}(2)$ fields requires two types of the fermions; one is the doublet fields under an $\mathrm{SU}(2)$, the others are doublet under the other SU(2). Some linear combinations of them are the SM left-handed fermions, and the other linear combinations are extra fermions. Therefore, if we add only one extra $\mathrm{SU}(2)$, then the symmetry to stabilize the dark matter requires to double the fermion fields compared to the SM. On the other hand, by considering two extra SU(2) gauge symmetries, we can realize the simple Yukawa interaction terms without extending the fermion sector as in eq. (2.8). This is a distinctive feature of this model from other $\mathrm{SU}(2)$ dark matter models. 


\subsection{Bosonic sector}

We briefly describe the electroweak sector and the related scalar sector. More details are discussed in appendices. The Lagrangian for those two sectors is given by

$$
\begin{aligned}
\mathcal{L} \supset & -\frac{1}{4} B_{\mu \nu} B^{\mu \nu}-\sum_{j=0}^{2} \sum_{a=1}^{3} \frac{1}{4} W_{j \mu \nu}^{a} W_{j}^{a \mu \nu} \\
& +D_{\mu} H^{\dagger} D^{\mu} H+\frac{1}{2} \operatorname{tr} D_{\mu} \Phi_{1}^{\dagger} D^{\mu} \Phi_{1}+\frac{1}{2} \operatorname{tr} D_{\mu} \Phi_{2}^{\dagger} D^{\mu} \Phi_{2}-V_{\text {scalar }}
\end{aligned}
$$

where

$$
\begin{aligned}
V_{\text {scalar }}= & m^{2} H^{\dagger} H+m_{\Phi}^{2} \operatorname{tr}\left(\Phi_{1}^{\dagger} \Phi_{1}\right)+m_{\Phi}^{2} \operatorname{tr}\left(\Phi_{2}^{\dagger} \Phi_{2}\right) \\
& +\lambda\left(H^{\dagger} H\right)^{2}+\lambda_{\Phi}\left(\operatorname{tr}\left(\Phi_{1}^{\dagger} \Phi_{1}\right)\right)^{2}+\lambda_{\Phi}\left(\operatorname{tr}\left(\Phi_{2}^{\dagger} \Phi_{2}\right)\right)^{2} \\
& +\lambda_{h \Phi} H^{\dagger} H \operatorname{tr}\left(\Phi_{1}^{\dagger} \Phi_{1}\right)+\lambda_{h \Phi} H^{\dagger} H \operatorname{tr}\left(\Phi_{2}^{\dagger} \Phi_{2}\right)+\lambda_{12} \operatorname{tr}\left(\Phi_{1}^{\dagger} \Phi_{1}\right) \operatorname{tr}\left(\Phi_{2}^{\dagger} \Phi_{2}\right) .
\end{aligned}
$$

Some coupling constants in the Higgs potential are common because of the discrete symmetry. We assume that the Higgs fields obtain the following vacuum expectation values at the global minimum.

$$
\langle H\rangle=\left(\begin{array}{c}
0 \\
\frac{v}{\sqrt{2}}
\end{array}\right), \quad\left\langle\Phi_{1}\right\rangle=\left\langle\Phi_{2}\right\rangle=\frac{1}{\sqrt{2}}\left(\begin{array}{cc}
v_{\Phi} & 0 \\
0 & v_{\Phi}
\end{array}\right) .
$$

The component fields of these Higgs fields at this vacuum are given by

$$
H=\left(\begin{array}{c}
i \pi_{3}^{+} \\
\frac{v+\sigma_{3}-i \pi_{3}^{0}}{\sqrt{2}}
\end{array}\right), \quad \Phi_{j}=\left(\begin{array}{cc}
\frac{v_{\Phi}+\sigma_{j}+i \pi_{j}^{0}}{\sqrt{2}} & i \pi_{j}^{+} \\
i \pi_{j}^{-} & \frac{v_{\Phi}+\sigma_{j}-i \pi_{j}^{0}}{\sqrt{2}}
\end{array}\right) .
$$

From the stationary condition, we find

$$
\begin{aligned}
m^{2} & =-\lambda v^{2}-2 \lambda_{h \Phi} v_{\Phi}^{2} \\
m_{\Phi}^{2} & =-\frac{\lambda_{h \Phi}}{2} v^{2}-\left(\lambda_{12}+2 \lambda_{\Phi}\right) v_{\Phi}^{2} .
\end{aligned}
$$

\subsection{Gauge sector}

After the electroweak symmetry breaking, the gauge boson mass terms are given by

$$
\left(\begin{array}{lll}
W_{0 \mu}^{+} & W_{1 \mu}^{+} & W_{2 \mu}^{+}
\end{array}\right) \mathcal{M}_{C}^{2}\left(\begin{array}{c}
W_{0}^{-\mu} \\
W_{1}^{-\mu} \\
W_{2}^{-\mu}
\end{array}\right)+\frac{1}{2}\left(\begin{array}{llll}
W_{0 \mu}^{3} & W_{1 \mu}^{3} & W_{2 \mu}^{3} & B_{\mu}
\end{array}\right) \mathcal{M}_{N}^{2}\left(\begin{array}{c}
W_{0}^{3 \mu} \\
W_{1}^{3 \mu} \\
W_{2}^{3 \mu} \\
B^{\mu}
\end{array}\right)
$$


where

$$
\begin{aligned}
\mathcal{M}_{C}^{2}= & \frac{1}{4}\left(\begin{array}{ccc}
g_{0}^{2} v_{\Phi}^{2} & -g_{0} g_{1} v_{\Phi}^{2} & 0 \\
-g_{0} g_{1} v_{\Phi}^{2} & g_{1}^{2}\left(v^{2}+2 v_{\Phi}^{2}\right) & -g_{1} g_{0} v_{\Phi}^{2} \\
0 & -g_{1} g_{0} v_{\Phi}^{2} & g_{0}^{2} v_{\Phi}^{2}
\end{array}\right), \\
\mathcal{M}_{N}^{2}= & \frac{1}{4}\left(\begin{array}{cccc}
g_{0}^{2} v_{\Phi}^{2} & -g_{0} g_{1} v_{\Phi}^{2} & 0 & 0 \\
-g_{0} g_{1} v_{\Phi}^{2} & g_{1}^{2}\left(v^{2}+2 v_{\Phi}^{2}\right) & -g_{1} g_{0} v_{\Phi}^{2} & -g_{1} g^{\prime} v^{2} \\
0 & -g_{1} g_{0} v_{\Phi}^{2} & g_{0}^{2} v_{\Phi}^{2} & 0 \\
0 & -g_{1} g^{\prime} v^{2} & 0 & g^{\prime 2} v^{2}
\end{array}\right) .
\end{aligned}
$$

After diagonalizing these mass matrices, we find the following mass eigenstates,

$$
\gamma, W^{ \pm}, Z, V^{0}, V^{ \pm}, W^{\prime \pm}, Z^{\prime}
$$

where $\gamma, W^{ \pm}$, and $Z$ are identified as the SM electroweak gauge bosons. $V^{0}$ and $V^{ \pm}$are odd under the discrete symmetry and are given by

$$
\begin{aligned}
V^{0} & =\frac{W_{0 \mu}^{3}-W_{2 \mu}^{3}}{\sqrt{2}}, \\
V^{ \pm} & =\frac{W_{0 \mu}^{ \pm}-W_{2 \mu}^{ \pm}}{\sqrt{2}} .
\end{aligned}
$$

The details, such as linear combinations for other gauge fields, are discussed in appendix A.

The masses of dark matter $V^{0}$ and its charged partner $V^{ \pm}$are given by

$$
m_{V^{ \pm}}^{2}=m_{V^{0}}^{2}=\frac{g_{0}^{2} v_{\Phi}^{2}}{4} \equiv m_{V}^{2},
$$

at the tree level. At the loop level, the mass difference is generated, and $m_{V^{ \pm}}$becomes slightly heavier than $m_{V^{0}}$ as we discuss in section 4.1. Therefore, $V^{0}$ is a dark matter candidate in our model.

\subsection{Physical scalars}

There are 12 scalars in the model, and 9 of them are would-be NG bosons. The three remaining neutral scalars are physical, and their mass terms are given by

$$
\mathcal{L} \supset \frac{1}{2}\left(\begin{array}{lll}
\sigma_{3} & \sigma_{1} & \sigma_{2}
\end{array}\right)\left(\begin{array}{ccc}
2 \lambda v^{2} & 2 v v_{\Phi} \lambda_{h \Phi} & 2 v v_{\Phi} \lambda_{h \Phi} \\
2 v v_{\Phi} \lambda_{h \Phi} & 8 v_{\Phi}^{2} \lambda_{\Phi} & 4 v_{\Phi}^{2} \lambda_{12} \\
2 v v_{\Phi} \lambda_{h \Phi} & 4 v_{\Phi}^{2} \lambda_{12} & 8 v_{\Phi}^{2} \lambda_{\Phi}
\end{array}\right)\left(\begin{array}{l}
\sigma_{3} \\
\sigma_{1} \\
\sigma_{2}
\end{array}\right) .
$$

After diagonalizing this mass matrix, we obtain the mass eigenstates, $h, h^{\prime}$, and $h_{D}$, where $h_{D}$ is odd under the discrete symmetry.

$$
\left(\begin{array}{c}
\sigma_{3} \\
\sigma_{1} \\
\sigma_{2}
\end{array}\right)=\left(\begin{array}{ccc}
\cos \phi_{h} & -\sin \phi_{h} & 0 \\
\frac{1}{\sqrt{2}} \sin \phi_{h} & \frac{1}{\sqrt{2}} \cos \phi_{h} & \frac{1}{\sqrt{2}} \\
\frac{1}{\sqrt{2}} \sin \phi_{h} & \frac{1}{\sqrt{2}} \cos \phi_{h} & -\frac{1}{\sqrt{2}}
\end{array}\right)\left(\begin{array}{c}
h \\
h^{\prime} \\
h_{D}
\end{array}\right) .
$$


If we choose the mass eigenvalues and the mixing angle $\left(m_{h}, m_{h^{\prime}}, m_{h_{D}}, \phi_{h}\right)$ as input parameters, then the quartic couplings in the Higgs potential are given by

$$
\begin{aligned}
\lambda & =\frac{m_{h}^{2} \cos ^{2} \phi_{h}+m_{h^{\prime}}^{2} \sin ^{2} \phi_{h}}{2 v^{2}}, \\
\lambda_{h \Phi} & =-\frac{\sin \phi_{h} \cos \phi_{h}}{2 \sqrt{2} v v_{\Phi}}\left(m_{h^{\prime}}^{2}-m_{h}^{2}\right), \\
\lambda_{\Phi} & =\frac{m_{h}^{2} \sin ^{2} \phi_{h}+m_{h^{\prime}}^{2} \cos ^{2} \phi_{h}+m_{h_{D}}^{2}}{16 v_{\Phi}^{2}}, \\
\lambda_{12} & =\frac{m_{h}^{2} \sin ^{2} \phi_{h}+m_{h^{\prime}}^{2} \cos ^{2} \phi_{h}-m_{h_{D}}^{2}}{8 v_{\Phi}^{2}} .
\end{aligned}
$$

\subsection{Model parameters}

The Lagrangian in the electroweak sector contains the following parameters.

$$
\left(g_{0}, g_{1}, g^{\prime}, m^{2}, m_{\Phi}^{2}, \lambda, \lambda_{\Phi}, \lambda_{h \Phi}, \lambda_{12}\right) .
$$

Instead of them, we can use the following parameters as inputs,

$$
\left(e, m_{Z}, v, m_{h}, m_{Z^{\prime}}, m_{V}, m_{h^{\prime}}, m_{h_{D}}, \phi_{h}\right),
$$

where $e$ is the QED coupling constant, and $v$ is related to the Fermi constant as

$$
v=\left(\sqrt{2} G_{F}\right)^{-1 / 2} .
$$

The first four parameters are already measured, and thus we have five free parameters in this model. The relation between the gauge couplings and the masses of the gauge bosons is discussed in appendix A. The derivation of eq. (2.30) is discussed in appendix C.

The analytical expression of the relations between eqs. (2.28) and (2.29) is complicated. In the following analysis, we numerically obtain the parameters in eq. (2.28) from a given set of parameters in eq. (2.29). However, in some limits, these relations can be simplified. Here we briefly show approximated expressions of some couplings for $v_{\Phi} \gg v$ that is typically realized for $m_{Z^{\prime}} \gg m_{Z}$. The approximate expressions help to understand the qualitative features of the model.

We introduce $g_{W}$ as

$$
g_{W} \equiv\left(\frac{2}{g_{0}^{2}}+\frac{1}{g_{1}^{2}}\right)^{-1 / 2}
$$

We find $g_{W} \simeq 0.65$ for $v_{\Phi} \gg v$ numerically, namely $g_{W}$ is approximately the $\mathrm{SU}(2)_{L}$ gauge coupling in the SM. Using $g_{W}, m_{Z^{\prime}}$, and $m_{V}$, we can obtain $g_{0}$, and $g_{1}$ as

$$
\begin{aligned}
g_{0} & \simeq \sqrt{2} g_{W} \frac{m_{Z^{\prime}}}{m_{V}} \frac{1}{\sqrt{\frac{m_{Z^{\prime}}^{2}}{m_{V}^{2}}-1}}, \\
g_{1} & \simeq g_{W} \frac{m_{Z^{\prime}}}{m_{V}} .
\end{aligned}
$$


The mass ratio of $Z^{\prime}$ and $V$ is given by

$$
\frac{m_{Z^{\prime}}^{2}}{m_{V}^{2}} \simeq 1+\frac{2 g_{1}^{2}}{g_{0}^{2}} .
$$

This equation shows that $m_{Z^{\prime}}>m_{V}$. Using these approximations, we obtain the masses of $W$ and $W^{\prime}$ as

$$
\begin{gathered}
m_{W}^{2} \simeq \frac{g_{W}^{2} v^{2}}{4} \\
m_{W^{\prime}}^{2} \simeq m_{Z^{\prime}}^{2}
\end{gathered}
$$

The gauge boson couplings to the fermions are given by

$$
\begin{aligned}
& g_{W u_{L} d_{L}}=g_{W \ell_{L} \nu_{L}}\left(\equiv g_{W f_{L} f_{L}}\right) \simeq g_{W}, \\
& g_{W^{\prime} u_{L} d_{L}}=g_{W^{\prime} \ell_{L} \nu_{L}}\left(\equiv g_{W^{\prime} f_{L} f_{L}}\right) \simeq-g_{W} \sqrt{\frac{m_{Z^{\prime}}^{2}}{m_{V}^{2}}-1,} \\
& g_{Z q_{L} q_{L}}=g_{Z \ell_{L} \ell_{L}}=g_{Z \nu_{L} \nu_{L}} \simeq \frac{e}{s_{Z} c_{Z}}\left(t^{3}-s_{Z}^{2} Q\right), \\
& g_{Z^{\prime} q_{L} q_{L}}=g_{Z^{\prime} \nu_{L} \ell_{L}}=g_{Z^{\prime} \nu_{L} \nu_{L}} \simeq-t^{3} g_{W} \sqrt{\frac{m_{Z^{\prime}}^{2}}{m_{V}^{2}}-1,} \\
& g_{Z q_{R} q_{R}}=g_{Z \ell_{R} \ell_{R}}=g_{Z \nu_{R} \nu_{R}} \simeq-\frac{e s_{Z}}{c_{Z}} Q, \\
& g_{Z^{\prime} q_{R} q_{R}}=g_{Z^{\prime} \ell_{R} \ell_{R}}=g_{Z^{\prime} \nu_{R} \nu_{R}}=\mathcal{O}\left(\frac{v^{2}}{v_{\Phi}^{2}}\right),
\end{aligned}
$$

where $t^{3}=\frac{1}{2}\left(-\frac{1}{2}\right)$ for up-type (down-type) fermions, $Q$ is the QED charge of the fermions, $c_{Z}=\sqrt{1-s_{Z}^{2}}$, and $s_{Z}$ is given as a solution of

$$
s_{Z}^{2} c_{Z}^{2}=\frac{v^{2} e^{2}}{4 m_{Z}^{2}} .
$$

We can see that the $W^{\prime}$ and $Z^{\prime}$ couplings to the SM fermions are controlled by the mass ratio of $Z^{\prime}$ and $V$. If $m_{Z^{\prime}}$ and $m_{V}$ are degenerated, then those couplings are suppressed while $g_{0}$ becomes very large. Therefore, we expect that the values of $W^{\prime}$ and $Z^{\prime}$ couplings to the SM fermions are comparable to those of the $W$ couplings in the region where perturbation works. We discuss this point further in section 3.2.

Using $g_{W}$ and the masses of the gauge bosons, we find that the triple gauge couplings are given by

$$
\begin{aligned}
g_{W W Z} \simeq g_{W^{\prime} W^{\prime} Z} \simeq g_{V^{-} V^{+} Z} & \simeq g_{W} \frac{m_{W}}{m_{Z}} \simeq g_{W W Z}^{\mathrm{SM}}, \\
g_{W W Z^{\prime}} & \simeq g_{W} \frac{m_{W}^{2}}{m_{Z^{\prime}}^{2}} \sqrt{\frac{m_{Z^{\prime}}^{2}}{m_{V}^{2}}-1,} \\
g_{W W^{\prime} Z} & \simeq g_{W} \frac{m_{W} m_{Z}}{m_{W^{\prime}}^{2}} \sqrt{\frac{m_{Z^{\prime}}^{2}}{m_{V}^{2}}-1,}
\end{aligned}
$$




$$
\begin{gathered}
g_{W^{\prime} W^{\prime} Z^{\prime}} \simeq g_{W} \frac{1}{\sqrt{\frac{m_{Z^{\prime}}^{2}}{m_{V}^{2}}-1}}\left(2-\frac{m_{Z^{\prime}}^{2}}{m_{V}^{2}}\right), \\
g_{W W^{\prime} Z^{\prime}}=g_{W^{+} V^{-} V^{0}}=g_{W^{-} V^{+} V^{0}} \simeq g_{W} \\
g_{V^{-} W^{\prime}+V^{0}}=g_{W^{\prime}-V^{+} V^{0}} \simeq g_{V^{-} V^{+} Z^{\prime}} \simeq g_{W} \frac{1}{\sqrt{\frac{m_{Z^{\prime}}^{2}}{m_{V}^{2}}-1}} .
\end{gathered}
$$

We emphasize that $V^{0}$ and $V^{ \pm}$couple to $W$ and $Z$ without any suppression factors, see eqs. (2.44) and (2.48). Therefore, DM pairs can annihilate into the SM gauge bosons through these couplings, $g_{V^{-} V^{+} Z}$ and $g_{W^{ \pm} V^{\mp} V^{0}}$. This is a distinctive feature of our vector DM model.

Couplings of physical scalar bosons to the gauge bosons are

$$
\begin{aligned}
g_{W W h} & \simeq \frac{2 m_{W}^{2}}{v} \cos \phi_{h} \simeq g_{W W h}^{\mathrm{SM}} \cos \phi_{h}, \\
g_{W W^{\prime} h} & \simeq \frac{2 m_{W}^{2}}{v}\left(-\cos \phi_{h} \sqrt{\frac{m_{Z^{\prime}}^{2}}{m_{V}^{2}}-1}+\frac{m_{W}}{m_{V}} \frac{m_{Z^{\prime}}}{m_{V}} \sin \phi_{h}\right), \\
g_{Z Z h} & \simeq \frac{2 m_{Z}^{2}}{v} \cos \phi_{h} \simeq g_{Z Z h}^{\mathrm{SM}} \cos \phi_{h}, \\
g_{Z Z^{\prime} h} & \simeq \frac{2 m_{W} m_{Z}}{v}\left(-\cos \phi_{h} \sqrt{\frac{m_{Z^{\prime}}^{2}}{m_{V}^{2}}-1}+\frac{m_{W}}{m_{V}} \frac{m_{Z^{\prime}}}{m_{V}} \sin \phi_{h}\right), \\
g_{V^{0} V^{0} h} \simeq g_{V^{+} V^{-} h} & \simeq \frac{g_{W} m_{Z^{\prime}}}{\sqrt{\frac{m_{Z^{\prime}}^{2}}{m_{V}^{2}}-1} \sin \phi_{h},} \\
g_{V^{0} V^{0} h^{\prime}} \simeq g_{V^{+} V^{-} h^{\prime}} & \simeq \frac{g_{W} m_{Z^{\prime}}}{\sqrt{\frac{m_{Z^{\prime}}^{2}}{m_{V}^{2}}}} \cos \phi_{h} .
\end{aligned}
$$

Note that $g_{W W h}$ is the same as the SM prediction for $\cos \phi_{h}=1$. This $g_{W W h}$ coupling is already measured by the ATLAS and CMS experiments, and the measured value is consistent with the SM value. Accordingly, we take small $\phi_{h}$ in the following analysis. For a small $\phi_{h}$ limit, the $V^{0}$ coupling to $h$ is suppressed. However, as we mentioned already, the annihilation processes of DM pairs into the SM particles do not need to rely on the DM-Higgs coupling. Therefore, we can obtain the right amount of DM energy density however small $\left|\phi_{h}\right|$ we take.

\section{Constraints}

\subsection{Perturbative unitarity}

We obtain the constraints on $g_{0}, g_{1}$, and scalar quartic couplings from the perturbative unitarity conditions for two-particle scattering processes in the high energy regime.

First, we consider two-to-two scalar bosons scattering processes in the high energy limit and derive the constraints on the scalar quartic couplings. In our derivation, we assume that 
these quartic couplings are much larger than the other couplings, such as gauge couplings. This model contains 12 scalars and there are 76 two scalar particle channels. We obtain the following conditions.

$$
\begin{array}{r}
|\lambda| \leq 4 \pi \\
\left|\lambda_{h \Phi}\right| \leq 4 \pi \\
\left|\lambda_{\Phi}\right| \leq \pi \\
\left|\lambda_{12}\right| \leq 2 \pi \\
\left|3 \lambda_{\Phi}-\lambda_{12}\right| \leq \pi \\
\left|3 \lambda+4\left(3 \lambda_{\Phi}+\lambda_{12}\right) \pm \sqrt{\left(3 \lambda-4\left(3 \lambda_{\Phi}+\lambda_{12}\right)\right)^{2}+32 \lambda_{h \Phi}^{2}}\right| \leq 8 \pi .
\end{array}
$$

Second, we can derive the upper bounds on the gauge couplings from vector-vector to scalar-scalar scattering processes. In our model, one of $g_{0}$ and $g_{1}$ can be larger than the other in most of the region of the parameter space, and thus the result in ref. [41] is applicable. We find that

$$
g_{j}<\sqrt{\frac{16 \pi}{\sqrt{6}}} \simeq 4.53 . \quad(j=0,1)
$$

\subsection{The mass ratio of $Z^{\prime}$ and $V$}

We find in section 2.4 that the mass ratio of $Z^{\prime}$ and $V$ is important to determine the model parameters and couplings. Although the mass ratio is a free parameter, there is a viable range.

It can be seen from eq. (2.32) that $g_{0}$ becomes very large for $m_{Z^{\prime}} \sim m_{V}$, and we can not treat $g_{0}$ as a small perturbation. For $m_{Z^{\prime}} \gg m_{V}$, we can see from eqs. (2.33) and (2.38) that $g_{1}$ and $g_{W^{\prime} f_{L} f_{L}}$ become large. This is also bad for the perturbative calculation. Moreover, the decay width of $W^{\prime}$ and $Z^{\prime}$ becomes larger for the larger $g_{W^{\prime} f_{L} f_{L}}$.

For $v_{\Phi} \gg v$ and $|\phi| \ll 1$, we find

$$
\begin{aligned}
\Gamma\left(W^{\prime} \rightarrow f \bar{f}\right) & \simeq \frac{N_{c}}{48 \pi} m_{W^{\prime}} g_{W f_{L} f_{L}}^{2}\left(\frac{m_{Z^{\prime}}^{2}}{m_{V}^{2}}-1\right), \\
\Gamma\left(W^{\prime} \rightarrow W Z\right) & \simeq \frac{1}{192 \pi} m_{W^{\prime}} g_{W f_{L} f_{L}}^{2}\left(\frac{m_{Z^{\prime}}^{2}}{m_{V}^{2}}-1\right), \\
\Gamma\left(W^{\prime} \rightarrow W h\right) & \simeq \frac{1}{192 \pi} m_{W^{\prime}} g_{W f_{L} f_{L}}^{2}\left(\frac{m_{Z^{\prime}}^{2}}{m_{V}^{2}}-1\right),
\end{aligned}
$$

where $N_{c}=3$ for quarks and 1 for leptons. Here we take $V_{C K M}=1$ for simplicity. If $W^{\prime}$ cannot decay into the non-SM particles kinematically, then the total width of $W^{\prime}$ is given by

$$
\Gamma_{W^{\prime}} \simeq m_{W^{\prime}} \frac{25}{96 \pi} g_{W}^{2}\left(\frac{m_{Z^{\prime}}^{2}}{m_{V}^{2}}-1\right)
$$




\begin{tabular}{|ccccc|}
\hline$m_{Z^{\prime}} / m_{V}$ & $g_{0}$ & $g_{1}$ & $\Gamma_{W^{\prime}} / m_{W^{\prime}}$ & $\left|g_{W^{\prime} f_{L} f_{L}} / g_{W f_{L} f_{L}}\right|$ \\
\hline 1.02 & 4.53 & 0.661 & 0.00148 & 0.207 \\
1.05 & 3 & 0.680 & 0.00358 & 0.321 \\
$\sqrt{2}$ & 1.30 & 0.916 & 0.0348 & 1 \\
4.63 & 0.938 & 3 & 0.711 & 4.52 \\
5.45 & 0.932 & 3.53 & 1 & 5.36 \\
6.97 & 0.93 & 4.53 & 1.66 & 6.90 \\
\hline
\end{tabular}

Table 2. The values of $g_{0}, g_{1}, \Gamma_{W^{\prime}} / m_{W^{\prime}}$, and $g_{W^{\prime} f_{L} f_{L}} / g_{W f_{L} f_{L}}$ given ratios of $m_{Z^{\prime}}$ and $m_{V}$.

We show some values of $g_{0}, g_{1}, \Gamma_{W^{\prime}} / m_{W^{\prime}}$, and $\left|g_{W^{\prime} f_{L} f_{L}} / g_{W f_{L} f_{L}}\right|$ for given ratios of $m_{Z^{\prime}}$ and $m_{V}$ in table 2. We find that we cannot treat $g_{0}$ as a small perturbation for $m_{Z^{\prime}} \simeq m_{V}$. We obtain a lower bound on the ratio of masses of $Z^{\prime}$ and $V$ as $m_{Z^{\prime}} / m_{V} \gtrsim 1.02$ from the perturbativity condition for $g_{0}$ shown in eq. (3.7). Similarly, the perturbativity for $g_{1}$ gives an upper bound on $m_{Z^{\prime}} / m_{V}$. We find $m_{Z^{\prime}} / m_{V}<6.97$. The total width also gives an upper bound on $m_{Z^{\prime}} / m_{V}$ because the total width is proportional to the imaginary part of the one-loop diagrams while the mass is at the tree level. Therefore, our calculation based on the perturbation is valid only for the region where $m_{W^{\prime}}>\Gamma_{W^{\prime}}$. This gives the upper bound on $m_{Z^{\prime}}$ for a given value of $m_{V}$, and we find that $m_{Z^{\prime}}<5.45 m_{V}$. We also find that $\Gamma_{W^{\prime}} / m_{W^{\prime}}<0.1$ is satisfied for $m_{W^{\prime}} \lesssim 2 m_{V}$.

\section{3 $W^{\prime}$ and $Z^{\prime}$ searches at the LHC}

New heavy vector bosons are being searched by the ATLAS and CMS experiments. Our model predicts the heavy vector bosons, $W^{\prime}$ and $Z^{\prime}$, and they couple to the SM particles. The $W^{\prime}$ and $Z^{\prime}$ couplings to SM particles are determined by the ratio of $m_{Z^{\prime}}$ and $m_{V}$ as discussed in section 2.4. The couplings to the fermions and the SM vector bosons can be as large as the $\mathrm{SU}(2)_{L}$ gauge coupling in the $\mathrm{SM}$, and the former is larger than the latter. Therefore, the main production process of $W^{\prime}$ and $Z^{\prime}$ at the LHC is $q \bar{q} \rightarrow W^{\prime} / Z^{\prime}$. The branching fraction to two fermions is larger than two bosons, see eqs. (3.8)-(3.10). Therefore, the main search channel of $W^{\prime}$ and $Z^{\prime}$ are $p p \rightarrow W^{\prime} \rightarrow \ell \nu$ and $p p \rightarrow Z^{\prime} \rightarrow \ell \ell$. The former gives the stronger constraint on the mass of $W^{\prime}$, and we focus on that process here.

The ATLAS experiment searches the $p p \rightarrow W^{\prime} \rightarrow \ell \nu$ process and finds the lower bound on $m_{W^{\prime}}$ as $6 \mathrm{TeV}$ for the Sequential Standard Model (SSM) [42]. ${ }^{4}$ The $W^{\prime}$ couplings to the SM fermions in our model are different from those in the SSM. We recast the bound and obtain the lower bound on $m_{W^{\prime}}$ for a given coupling ratio of $g_{W^{\prime} f_{L} f_{L}}$ and $g_{W f_{L} f_{L}}$. The result is shown in figure 1. Here we assume that the $K$ factor is 1.3 . We find that $m_{W^{\prime}} \gtrsim 7 \mathrm{TeV}$ for $g_{W^{\prime} f_{L} f_{L}} / g_{W f_{L} f_{L}} \gtrsim 1.42$. Since the ATLAS experiment does not give the bound for $m_{W^{\prime}}>7 \mathrm{TeV}$, we cannot obtain the bound on $m_{W^{\prime}}$ for $g_{W^{\prime} f_{L} f_{L}} / g_{W f_{L} f_{L}} \gtrsim 1.42$.

\footnotetext{
${ }^{4}$ The CMS experiment also searches the same channel but gives a weaker bound on $m_{W^{\prime}}, m_{W^{\prime}}>$ $5.2 \mathrm{TeV}[43]$.
} 


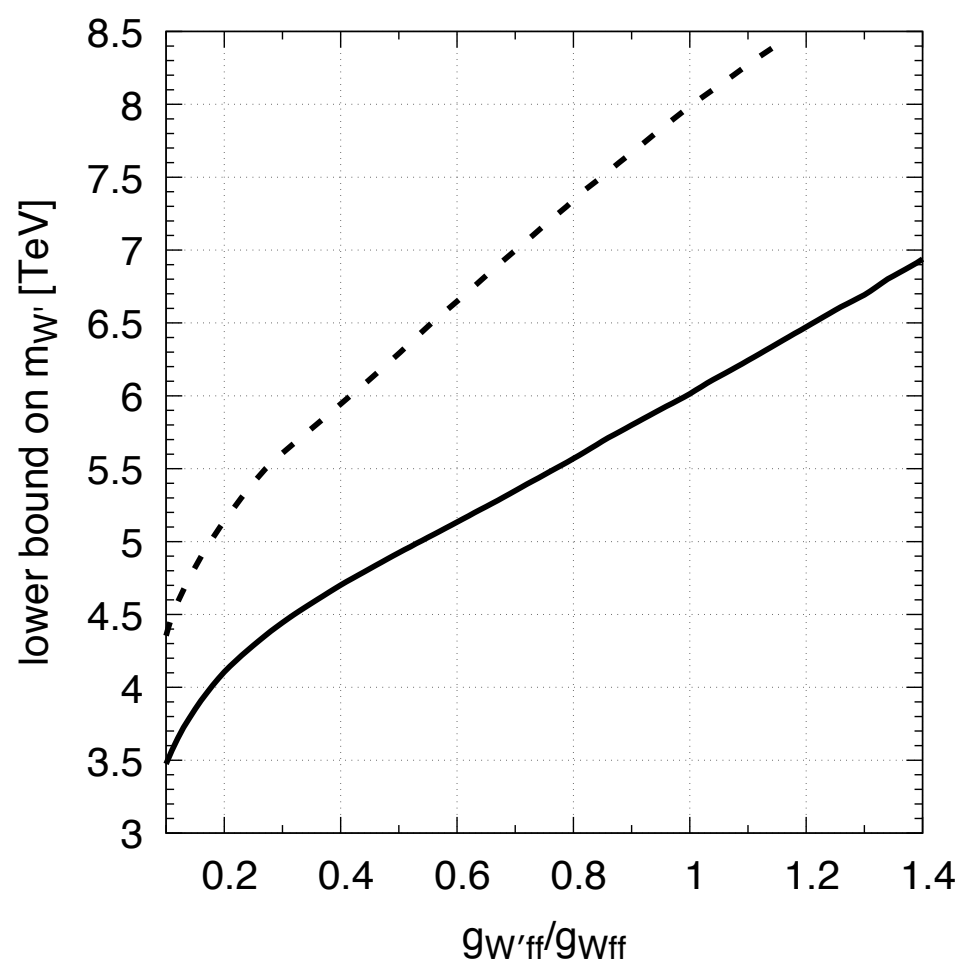

Figure 1. The solid curve shows the lower bound on $m_{W^{\prime}}$ for a given $g_{W^{\prime} f_{L} f_{L}}$ coupling obtained by recasting the result in ref. [42]. The dashed curve shows the prospect at the ATLAS experiment with $14 \mathrm{TeV}$ with $3000 \mathrm{fb}^{-1}[44]$.

Similarly, we also recast the prospect of $W^{\prime}$ search at the ATLAS experiment with $14 \mathrm{TeV}$ with $3000 \mathrm{fb}^{-1}$ [44]. Other channels give weaker bound than this $\ell \nu$ channel.

\subsection{Electroweak precision measurements}

For $m_{W^{\prime} / Z^{\prime}} \gg m_{W / Z}$ limit, it is easy to obtain the electroweak precision parameters, $\hat{S}, \hat{T}$, $W$, and $Y$, introduced in [45]. At the tree level, we find that

$$
\begin{aligned}
\hat{S} & =\hat{T}=Y=0, \\
W & =\frac{2 g_{1}^{2}}{g_{0}^{2}+2 g_{1}^{2}} \frac{m_{W}^{2}}{m_{W^{\prime}}^{2}} . \simeq\left(1-\frac{m_{V}^{2}}{m_{Z^{\prime}}^{2}}\right) \frac{m_{W}^{2}}{m_{W^{\prime}}^{2}} .
\end{aligned}
$$

The constraint is given as $W=(-0.3 \pm 0.6) \times 10^{-3}$. We find that this constraint is much weaker than the constraint from the $W^{\prime}$ search at the LHC experiment.

\subsection{Higgs signal strength}

Among the three scalar fields, only $H$ contributes to the Yukawa interaction terms, and thus the $h$ couplings to the fermions are equal to those in the SM times $\cos \phi_{h}$. As we have shown in eq. (2.50), $g_{W W h}$ for $v_{\Phi} \gg v$ is approximately given by the SM coupling times $\cos \phi_{h}$. Thus the Higgs signal strengths are given by

$$
\kappa_{F}=\cos \phi_{h}, \quad \kappa_{V} \simeq \cos \phi_{h} .
$$


We can constrain $\phi_{h}$ from the measurement of the Higgs couplings. We use the result from the ATLAS experiment [46],

$$
\begin{aligned}
& \kappa_{V}=1.05 \pm 0.04, \\
& \kappa_{F}=1.05 \pm 0.09,
\end{aligned}
$$

with the linear correlation between them is observed as $44 \%$, and obtain $\left|\phi_{h}\right|<0.3$. We consider $0 \leq\left|\phi_{h}\right|<0.3$ in the following discussions.

\section{DM phenomenology}

\subsection{Mass difference and its implication for collider physics}

At the tree level, $V^{0}$ and $V^{ \pm}$have the same mass. However, the mass difference is generated at the loop level, and thus $V^{ \pm}$is slightly heavier than $V^{0}$. The mass difference is given by

$$
\begin{aligned}
\delta_{m_{V}} \equiv m_{V^{ \pm}}-m_{V^{0}} & =\sqrt{m_{V}^{2}+\Pi_{V^{+} V^{-}}\left(m_{V^{ \pm}}^{2}\right)}-\sqrt{m_{V}^{2}+\Pi_{V^{0} V^{0}}\left(m_{V^{0}}^{2}\right)} \\
& \simeq \frac{\Pi_{V^{+} V^{-}}\left(m_{V}^{2}\right)-\Pi_{V^{0} V^{0}}\left(m_{V}^{2}\right)}{2 m_{V}},
\end{aligned}
$$

where $\Pi_{V^{+} V^{-}}$and $\Pi_{V^{0} V^{0}}$ are the self-energies of $V^{ \pm}$and $V^{0}$, respectively. We calculate $\delta_{m_{V}}$ at the one-loop level by using FormCalc [47]. In $v_{\Phi} \gg v$ limit, we find

$$
\delta_{m_{V}} \simeq \frac{m_{W}^{3} G_{F}}{\sqrt{2} \pi}\left(1-\frac{m_{W}}{m_{Z}}\right) \simeq 168 \mathrm{MeV}
$$

This result is consistent with the result in [48]. We have also checked it numerically by using LoopTools [47], without taking $v_{\Phi} \gg v$ limit.

This small mass difference is the same as the mass difference between the charged and neutral components of Wino $(\tilde{W})$ in the MSSM. Wino is $\mathrm{SU}(2)_{L}$ triplet fermions. The charged Wino decays into the neutral Wino, but its lifetime is long due to the small mass difference. Thus, Wino is being searched in the long-lived particle searches at the LHC. Our DM candidate, $V^{0}$, and its partner, $V^{ \pm}$, has the same properties as the Wino. The decay rate of $V^{ \pm}$and the mass difference of $V^{ \pm}$and $V^{0}$ are exactly equal to those of Wino. Therefore, the long-lived particle search is also a useful tool to find $V^{ \pm}$in our model. The only difference of $V$ from $\tilde{W}$ is the production rate of the charged particles. Figure 2 shows the production cross sections of $V^{ \pm}$and $\tilde{W}^{ \pm}$at the LHC with $\sqrt{s}=13 \mathrm{TeV}$. We find that the production cross section of $V^{ \pm, 0}$ depends on $m_{W^{\prime}}$ and $m_{Z^{\prime}}$ as well as $m_{V}$. It is also found that the production cross section of $V^{ \pm, 0}$ is smaller than the production cross section of Wino because of the interference between the diagrams exchanging $W$ and $W^{\prime}(Z$ and $\left.Z^{\prime}\right)$ in the $s$-channel. Therefore, the constraint on $m_{V}$ from the long-lived particle search is weaker than that on the Wino, $m_{\tilde{W}} \gtrsim 460 \mathrm{GeV}$ [49]. Once we require $V^{0}$ to explain the measured value of the DM energy density, then $m_{V} \gtrsim 3 \mathrm{TeV}$ is required as we will see in the following. Therefore, our model is consistent with the results of the long-lived search if the whole of DM in our universe is explained by $V^{0}$. 

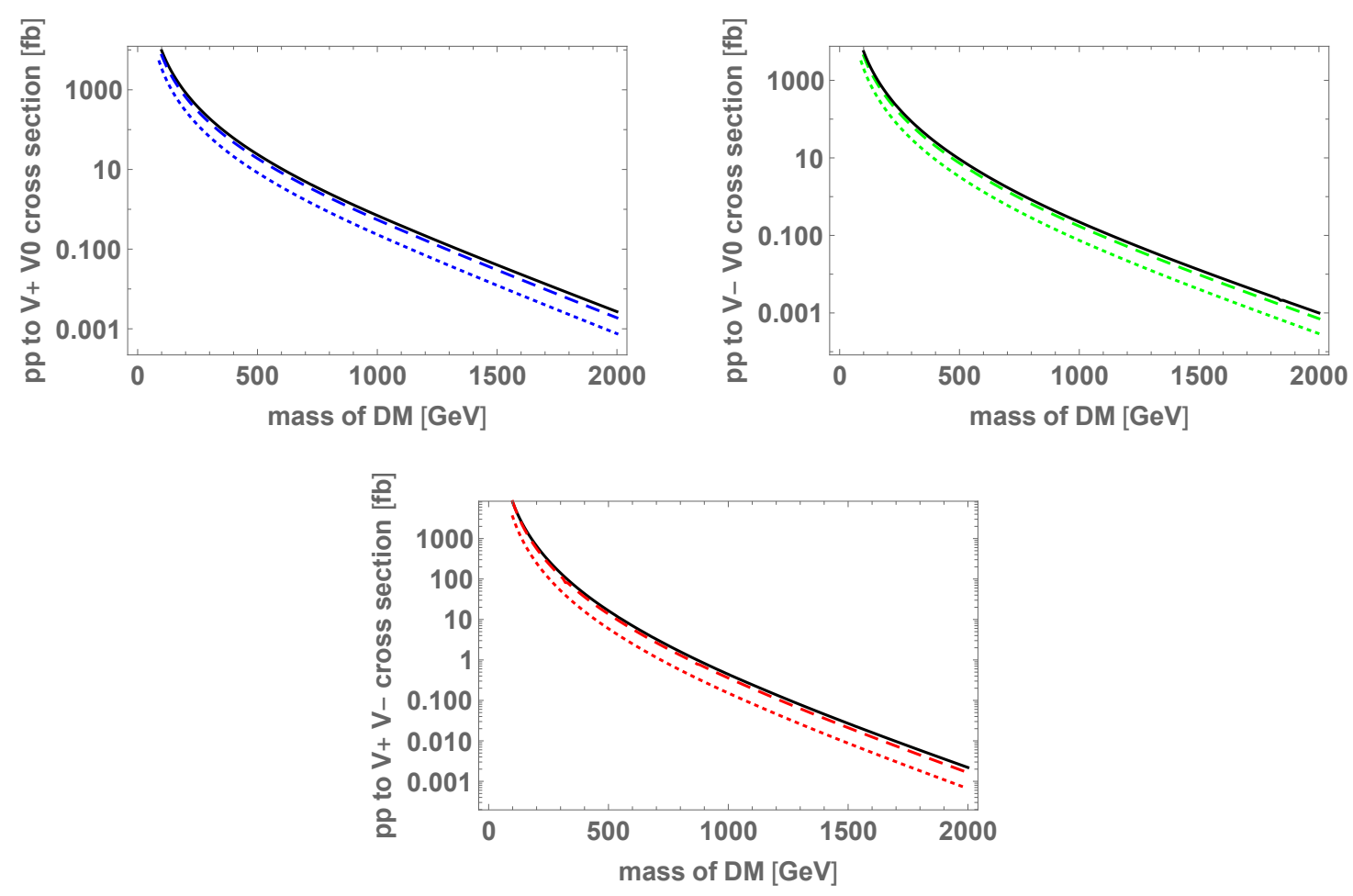

Figure 2. The production cross section of $V^{ \pm}$and $\tilde{W}^{ \pm}$from proton collisions at $\sqrt{s}=13 \mathrm{TeV}$. The left panel shows the cross section of $p p \rightarrow V^{+} V^{0}$, the right shows $p p \rightarrow V^{-} V^{0}$, and the bottom shows $p p \rightarrow V^{+} V^{-}$. In each figure, the black line shows the Wino production cross section and the dashed (dotted) line shows the $V^{ \pm}$for $m_{Z^{\prime}, W^{\prime}}=1.5 m_{V}\left(m_{Z^{\prime}, W^{\prime}}=1.3 m_{V}\right)$.

\subsection{Direct detection}

At the leading order, DM-nucleon scattering is mediated by two scalars, $h$ and $h^{\prime}$, which are even under the discrete symmetry. The spin-independent vector DM-nucleon scattering cross section is given by

$$
\sigma_{\mathrm{SI}}^{N}=\frac{1}{\pi}\left(\frac{m_{N}}{m_{N}+m_{V}}\right)^{2}\left|f_{N V}\right|^{2}
$$

where $m_{N}$ is the nucleon mass $(N=p, n)$ and $f_{N V}$ is the effective coupling of DM-nucleon interactions.

Figure 3 shows the leading diagrams at the parton-level. The following Parton-level effective interactions are relevant to the DM-nucleon cross section,

$$
\mathcal{L}^{\mathrm{eff}}=\sum_{q=u, d, s} c_{q} V^{0 \mu} V_{\mu}^{0} m_{q} \bar{q} q+\sum_{Q=c, b, t} c_{Q} V^{0 \mu} V_{\mu}^{0} m_{Q} \bar{Q} Q
$$

where $m_{q}$ and $m_{Q}$ are light and heavy quark masses, respectively. The couplings, $c_{q}$ and $c_{Q}$, in our model are

$$
c_{q}=c_{Q}=\frac{m_{V}^{2}}{\sqrt{2} v v_{\Phi}} \sin \phi_{h} \cos \phi_{h}\left(\frac{1}{m_{h^{\prime}}^{2}}-\frac{1}{m_{h}^{2}}\right) .
$$



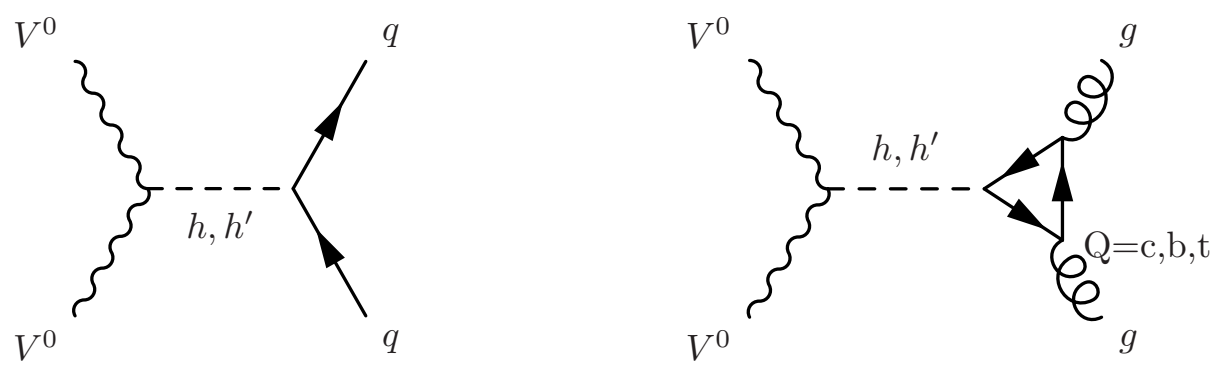

Figure 3. The leading diagrams mediated by $h$ and $h^{\prime}$.

To obtain the effective coupling of the DM-nucleon interactions, $f_{N V}$, we use the nucleon matrix elements,

$$
\begin{aligned}
\left\langle N\left|m_{q} \bar{q} q\right| N\right\rangle & \equiv m_{N} f_{T q}^{(N)}, \quad(q=u, d, s), \\
\left\langle N\left|\frac{\alpha_{s}}{\pi} G_{\mu \nu}^{a} G^{a \mu \nu}\right| N\right\rangle & =-\frac{8}{9} m_{N}\left(1-\sum_{q} f_{T q}^{(N)}\right) .
\end{aligned}
$$

where $G_{\mu \nu}^{a}$ and $\alpha_{s}$ are the $\mathrm{SU}(3)_{c}$ field strength tensor and coupling constant, respectively. The numerical values of the mass fractions for the nucleon, $f_{T q}^{(N)}(N=p, n)$, are obtained by lattice simulations, and we take the default values of micrOMEGAs [50].

$$
\begin{aligned}
f_{T u}^{p} & =0.0153, & & f_{T u}^{n}=0.011 \\
f_{T d}^{p} & =0.0191, & & f_{T d}^{n}=0.0273 \\
f_{T s}^{p} & =f_{T s}^{n}=0.0447 . & &
\end{aligned}
$$

For light quarks $(q=u, d, s)$, we can obtain the contribution to the effective coupling $f_{N V}$ using nucleon matrix elements of the mass operators. For the heavy quarks $(Q=c, b, t)$, the leading contribution is loop diagrams (figure 3 right). The operator $m_{Q} \bar{Q} Q$ equals $-\frac{\alpha_{s}}{12 \pi} G_{\mu \nu}^{a} G^{a \mu \nu}$ in the matrix element, so the matrix elements of the heavy quark mass operators are given by

$$
\left\langle N\left|m_{Q} \bar{Q} Q\right| N\right\rangle=\frac{2}{27} m_{N}\left(1-\sum_{q} f_{T q}^{(N)}\right), \quad(Q=c, b, t) .
$$

Using these matrix elements, the effective coupling $f_{N V}$ is given by

$$
\begin{aligned}
\frac{f_{N V}}{m_{N}} & =\sum_{q} c_{q} f_{T q}^{N}+\frac{2}{27} \sum_{Q} c_{Q}\left(1-\sum_{q} f_{T q}^{N}\right) \\
& =\frac{m_{V}^{2}}{\sqrt{2} v v_{\Phi}} \sin \phi_{h} \cos \phi_{h}\left(\frac{1}{m_{h^{\prime}}^{2}}-\frac{1}{m_{h}^{2}}\right)\left(\frac{2}{9}+\frac{7}{9} \sum_{q} f_{T q}^{(N)}\right) .
\end{aligned}
$$


Finally, we obtain the spin-independent nucleon-vector DM cross section as follows.

$$
\begin{aligned}
\sigma_{\mathrm{SI}}^{N} & =\frac{1}{2 \pi} \frac{m_{N}^{4} m_{V}^{4}}{\left(m_{N}+m_{V}\right)^{2}} \frac{1}{v^{2} v_{\Phi}^{2}} \sin ^{2} \phi_{h} \cos ^{2} \phi_{h}\left(\frac{1}{m_{h^{\prime}}^{2}}-\frac{1}{m_{h}^{2}}\right)^{2}\left(\frac{2}{9}+\frac{7}{9} \sum_{q=u, d, s} f_{T q}^{N}\right)^{2} \\
& \simeq \frac{g_{0}^{2}}{32 \pi v^{2}} \frac{m_{N}^{4}}{m_{h}^{4}} \sin ^{2}\left(2 \phi_{h}\right)\left(\frac{2}{9}+\frac{7}{9} \sum_{q=u, d, s} f_{T q}^{N}\right)^{2} \\
& \simeq 10^{-44} \times g_{0}^{2} \sin ^{2}\left(2 \phi_{h}\right)\left[\mathrm{cm}^{2}\right] .
\end{aligned}
$$

Here we assumed that $m_{V} \gg m_{N}$, and also $m_{h^{\prime}} \gg m_{h}$ in the last two lines of eq. (4.11). This cross section is proportional to $\sin ^{2}\left(2 \phi_{h}\right)$, and thus the large $\left|\phi_{h}\right|$ region is severely constrained from the direct detection experiments. The direct detection limit on the DMnucleon cross section for TeV scale DM is around $10^{-45} \mathrm{~cm}^{2}$ [3]. For $g_{0}=1$, we find $\phi_{h} \lesssim 0.15$. This upper bound can be stronger than the bound from the Higgs signal strength. If $\phi_{h}$ is smaller than $\sim 0.01$, the higher-order diagrams dominate in the DMnucleon SI scattering process so that $\sigma_{\mathrm{SI}}^{N} \sim 10^{-47} \mathrm{~cm}^{2}[51-53]$.

\subsection{Relic abundance}

The model contains two DM candidates, $V^{0}$ and $h_{D}$. In this paper, we treat $V^{0}$ as the DM candidate by assuming $h_{D}$ is always heavier than $V^{0}$.

We calculate the thermal relic abundance of $V^{0}$ by using micrOMEGAs [50]. The model file is generated by FeynRules [54]. Since the mass difference of $V^{ \pm}$and $V^{0}$ is tiny, the coannihilation processes, which are automatically calculated in micrOMEGAs, are relevant. All the masses of the new particles are proportional to $v_{\Phi}$, hence the large mass difference among the new particles requires large couplings. To avoid large couplings and to keep working within the perturbative regime, we keep the mass ratio of the new particles to the DM mass within $\mathcal{O}(1)$.

The vector DM can interact with the SM weak gauge bosons even in a limit of vanishing the scalar mixing $\phi_{h}$. We start by investigating the relic abundance with very small $\phi_{h}$ and show that the vector DM can explain the measured value of the DM energy density. We also discuss the case for $\left|\phi_{h}\right| \sim \mathcal{O}(0.1)$ to see the impact of $\phi_{h}$ on the forthcoming direct detection experiments.

\subsubsection{Very small $\left|\phi_{h}\right|$ case}

Figure 4 shows the DM relic abundance in an $m_{V}-m_{Z^{\prime}}$ plane for the very small $\left|\phi_{h}\right|$. We take $\phi_{h}=0.001$ here, and the same result is obtained for much smaller $\phi_{h}$. This is because the hidden vector bosons, $V^{0}$ and $V^{ \pm}$, efficiently annihilate into visible vector bosons and do not need to rely on $h$ and $h^{\prime}$ exchanging processes. The other new particle masses are fixed as $m_{h_{D}}=1.2 m_{V}$ and $m_{h^{\prime}}=1.4 m_{V}$. The result is insensitive to the choice of $m_{h_{D}}$ and $m_{h^{\prime}}, m_{h_{D}}=1.2 m_{V}$ and $m_{h^{\prime}}=1.4 m_{V}$. We find three viable regions of parameter space for the explanation of the measured value of the DM energy density [1] as a thermal 


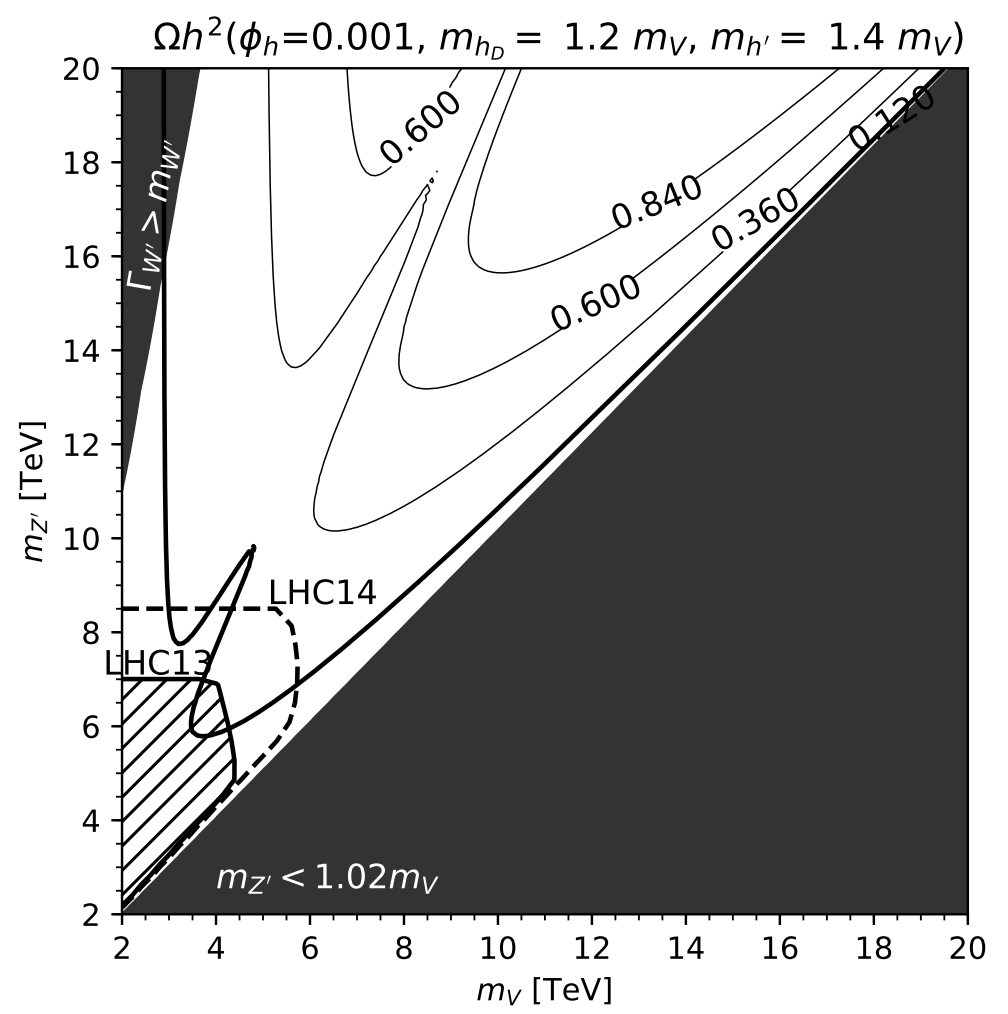

Figure 4. The contours show the DM relic abundance as a function of the masses of the DM and $Z^{\prime}$. Here, $\phi_{h}=0.001, m_{h_{D}}=1.2 m_{V}$ and $m_{h^{\prime}}=1.4 m_{V}$. The measured value of the DM energy density is shown by the thick-solid contour. The DM is overabundant in the region above the thicksolid contour. The region filled by the hatched pattern is excluded by the ATLAS experiment [42]. The prospect at the HL-LHC is also shown by the dashed curve [44]. In the larger black-shaded region, $g_{0}$ is beyond the perturbative unitarity bound, see table 2. In the black-shaded region in the left-top corner, $\Gamma_{W^{\prime}}>m_{W^{\prime}}$.

relic: the narrow $W^{\prime}$ width region $\left(m_{Z^{\prime}} \lesssim 2 m_{V}\right)$, the $V^{\prime}$-resonant region $\left(m_{Z^{\prime}} \simeq 2 m_{V}\right)$, and the wide $W^{\prime}$ width region $\left(m_{Z^{\prime}} \gtrsim 2 m_{V}\right)$.

For the narrow $W^{\prime}$ width region, pairs of the dark vector bosons mainly annihilate into visible massive gauge bosons including $W^{\prime \pm}$ and $Z^{\prime}$. In this region, we find $m_{V} \gtrsim 4.2 \mathrm{TeV}$ from the constraint on the $W^{\prime}$ search by the ATLAS experiment [42]. It is possible to test this case for $m_{V} \lesssim 5.8 \mathrm{TeV}$ by the $W^{\prime}$ search at the HL-LHC [44]. For the larger $m_{V}$, we can avoid the constraint from the $W^{\prime}$ and $Z^{\prime}$ search because it requires heavier $W^{\prime}$ and $Z^{\prime}$ to obtain the measured value of the DM energy density. However, it also requires the larger $g_{0}$, and thus the perturbative unitarity of $g_{0}$ gives the upper bound on $m_{V}$.

In the $V^{\prime}$-resonant region, which looks like a horn in the figure, the main (co)annihilation channel is $V^{0} V^{ \pm} \rightarrow q \bar{q}$ via $W^{\prime}$ exchange in the $s$-channel. In this region, $g_{0}$ and $g_{1}$ are less than $\mathcal{O}(1)$, and the perturbative unitarity is easily satisfied.

In the wide $W^{\prime}$ width region, pairs of the dark matter particles mainly annihilate into $W^{ \pm}$and $Z$ because the processes with a $W^{\prime \pm}$ or a $Z^{\prime}$ in final states are kinematically forbidden in this region. The masses of $W^{\prime}$ and $Z^{\prime}$ are larger than the dark matter particles, 
and thus $W^{\prime}$ and $Z^{\prime}$ are almost decoupled from the annihilation processes. As a result, $m_{V}$ is almost fixed around $3 \mathrm{TeV}$ if we demand $\Omega h^{2}=0.12$. This region is similar to the Wino $\mathrm{DM}$ model and $\mathrm{SU}(2)_{L}$ triplet scalar DM models [48, 55]. In those models, DM mainly annihilates into $W^{ \pm}$and $Z$, and the mass of the DM is fixed by requiring the thermal relic to explain the measured value of the DM energy density.

\subsubsection{For $\left|\phi_{h}\right| \sim \mathcal{O}(0.1)$}

We discuss the case for $\left|\phi_{h}\right| \simeq \mathcal{O}(0.1)$ to see the effects of $\phi_{h}$ to the thermal relic abundance and the direct detection experiments. In this regime, the scalar quartic couplings can be large with large $m_{h^{\prime}}$ as can be seen from eqs. (2.24)-(2.27). The annihilation processes into $h$ and $h^{\prime}$, which are proportional to the quartic couplings, are efficient, and $\phi_{h}$ dependence is visible.

Figure 5 shows the value of $\phi_{h}$ that is required to obtain the measured value of the DM energy density. Comparing to figure 4, the viable region that explains the right amount of DM relic abundance is extended. The larger $\phi_{h}$ requires the heavier $m_{Z^{\prime}}$. This is because $h$ and $h^{\prime}$ contribute to the annihilation of pairs of DM particles for larger $\phi_{h}$, and the contributions of $W^{\prime}$ and $Z^{\prime}$ have to be smaller. On the other hand, the region with the larger $\phi_{h}$ is excluded by the constraint on the SM Higgs couplings as we discussed in section 3.5. As a result, we can constrain the value of $m_{Z^{\prime}}$ for a given $m_{V}$.

We discuss the lighter and heavier $Z^{\prime}$ regions in detail. The left panel in figure 6 is for the heavier $Z^{\prime}$ region. It shows that the constraint from the XENON1T experiment is stronger than the one from the Higgs coupling measurements. We find that the XENONnT experiment [56] can cover most of the parameter space for $\phi_{h} \gtrsim \mathcal{O}(0.01)$. The constraint from the perturbative unitarity gives a stronger constraint than one from the XENON1T experiment. However, this constraint highly depends on the choice of $m_{h^{\prime}}$. The right panel in figure 6 is for the lighter $Z^{\prime}$ region. The XENONnT covers the large region of the parameter space. The HL-LHC is also useful to test the model for $m_{W^{\prime}}<5.7 \mathrm{TeV}$. The $W^{\prime}$ search at the collider experiment is independent of $\phi_{h}$, therefore the XENONnT experiment and the HL-LHC is complementary to each other.

The smaller $\phi_{h}$ region is degenerate in figures 5 and 6 . We magnify those regions in figure 7 . The values of $m_{Z^{\prime}}$ that are required to obtain the right amount of DM energy density are shown in the $m_{V^{-}} \phi_{h}$ plane. The left panel shows the lighter $m_{V}$ region. We find that the combination of the DM direct detection at the XENONnT experiment and the $W^{\prime}$ search at the HL-LHC is a powerful tool to seek this region. The former will give an upper bound on $\phi_{h}$ that is almost independent of $m_{V}$. The latter, on the other hand, is sensitive for $3 \mathrm{TeV} \lesssim m_{V} \lesssim 3.9 \mathrm{TeV}$. For the lighter $m_{V}, m_{V} \lesssim 3 \mathrm{TeV}$, the $W^{\prime}$ decay width can be as large as $m_{V}$, but in most of the region it satisfies $0.1<\Gamma_{W^{\prime}} / m_{W^{\prime}}<0.2$. The right panel in figure 7 is for $V$ that is heavier than $4 \mathrm{TeV}$. The direct detection experiment is important in this region as well to determine the value of $\phi_{h}$. For $m_{V} \lesssim 6 \mathrm{TeV}$, we can test this model from the $W^{\prime}$ search. We find that the perturbative unitarity of scalar quartic couplings gives the upper limit on $m_{V}, m_{V} \lesssim 19 \mathrm{TeV}$. 


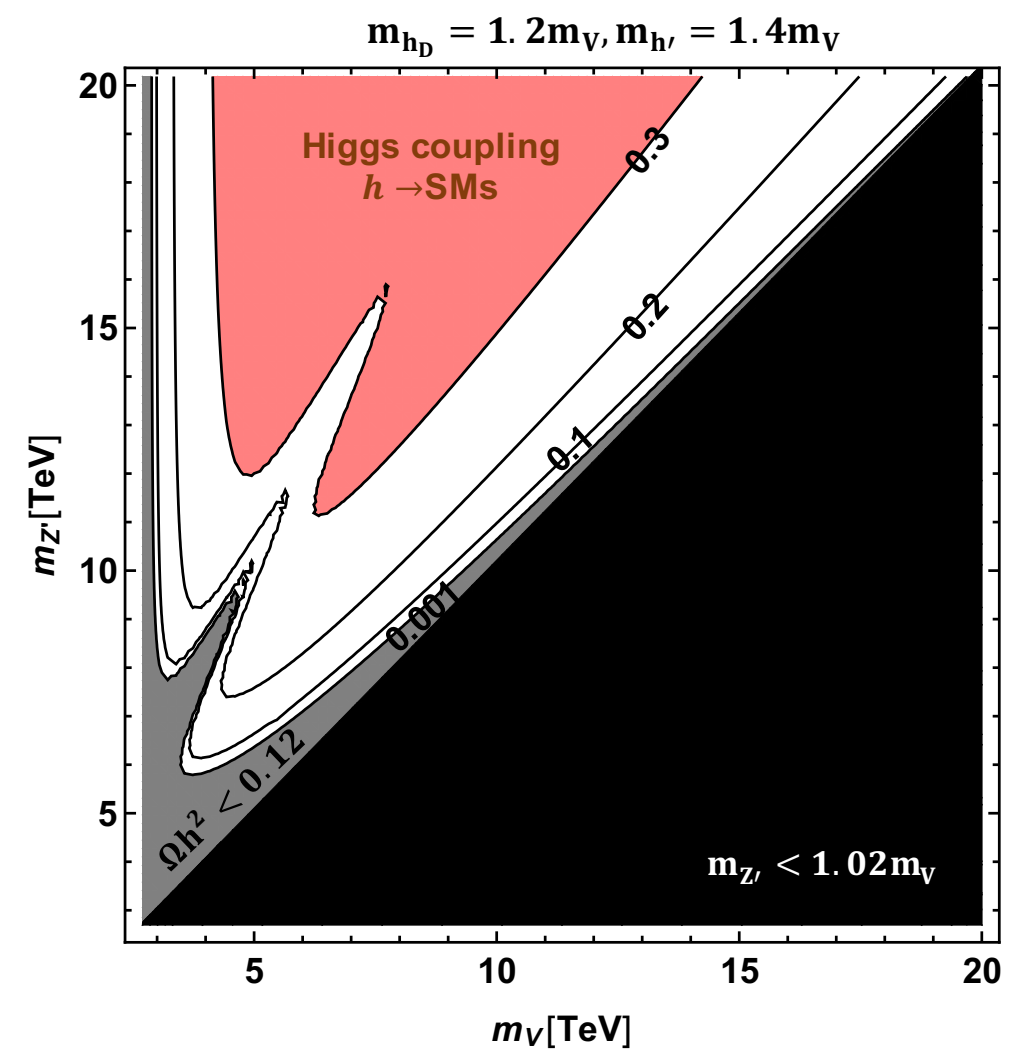

Figure 5. The contours show $\phi_{h}$ that reproduce the measured value of the DM relic abundance. Here, $m_{h_{D}}=1.2 m_{V}$ and $m_{h^{\prime}}=1.4 m_{V}$. In the gray shaded region, this model cannot explain the whole abundance. The pink region $\left(\phi_{h}>0.3\right)$ is constrained by the measurement of the Higgs signal strength [46]. In the black shaded region, $g_{0}$ is beyond the perturbative unitarity bound.

\section{Conclusions}

We constructed a model of spin-1 dark matter that has the electroweak gauge interaction. The electroweak gauge symmetry is extended into $\mathrm{SU}(2)_{0} \times \mathrm{SU}(2)_{1} \times \mathrm{SU}(2)_{2} \times \mathrm{U}(1)_{Y}$, and the discrete symmetry under the exchanging of $\mathrm{SU}(2)_{0}$ and $\mathrm{SU}(2)_{2}$ is imposed. It is not necessary to extend the fermion sector to realize the realistic fermion mass spectra through the Yukawa interactions. Since the dark matter candidate in this model couples to the electroweak gauge bosons, we do not need to rely on the Higgs portal couplings. These two features are distinctive of our model from other spin-1 dark matter models. Our model predicts spin- 0 and spin- 1 dark matter candidates, and the heavier one decays into the lighter one. In this paper, we focus on the spin-1 dark matter candidate.

The model predicts a heavy vector triplet $\left(W^{\prime \pm}\right.$ and $\left.Z^{\prime}\right)$ in the visible sector. We found that the $W^{\prime}$ searches at the LHC give a strong constraint. That has already excluded some regions of the parameter space that can explain the measured value of the dark matter energy density by the freeze-out mechanism.

There are three scenarios that the model predicts the right amount of the dark matter relic abundance. The first scenario is that the heavy vector triplet is slightly heavier than 

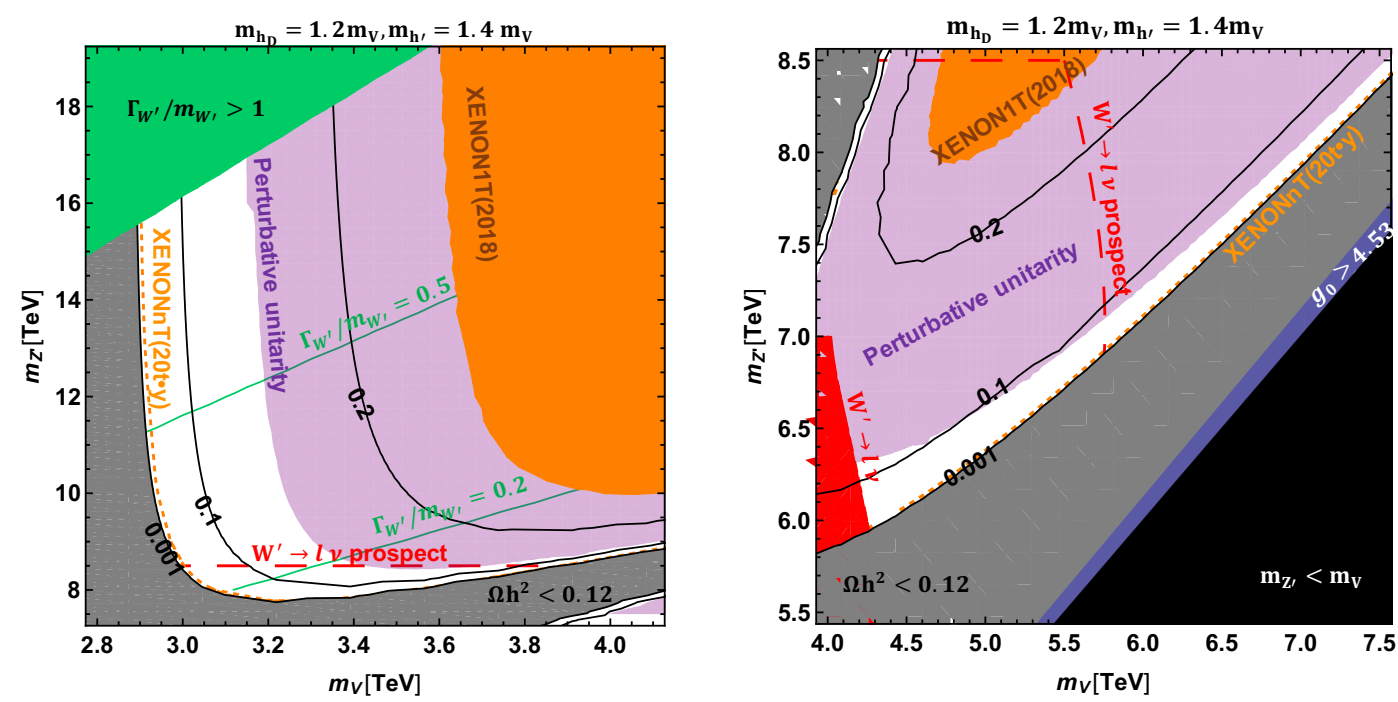

Figure 6. Combined results in the $m_{V}-m_{Z^{\prime}}$ plane. The gray shaded region cannot explain the whole abundance and the black shaded region is theoretically forbidden. The orange region is already constrained by the XENON2018 and the dotted line shows the prospect by XENONnT (20 ton-year). In the purple region, some of the Higgs quartic couplings are non-perturbative. The red shaded region is constrained by ATLAS and the red dashed line shows the prospect. In the left panel, the green line shows the value of $\Gamma_{W^{\prime}} / m_{W^{\prime}}$, and $\Gamma_{W^{\prime}}>m_{W^{\prime}}$ in the green shaded region. In the right panel, $g_{0}$ is beyond the perturbative unitarity bound in the blue shaded region.
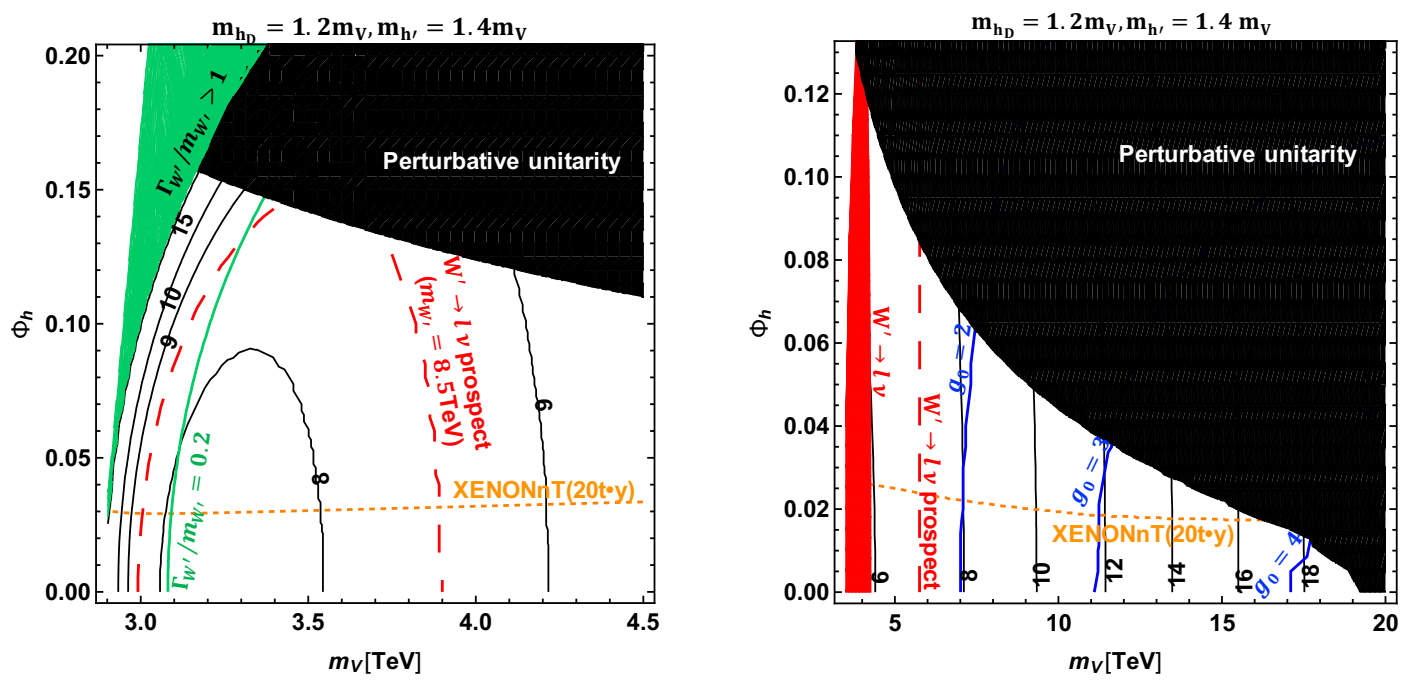

Figure 7. Combined results in the $m_{V}-\phi_{h}$ plane. The left (right) panel shows the case for $m_{Z^{\prime}}>$ $2 m_{V}\left(m_{Z^{\prime}}<2 m_{V}\right)$. We determine $m_{Z^{\prime}}$ to obtain the right amount of the DM relic abundance, and the values are shown by the black-solid contours in $\mathrm{TeV}$ unit. The orange dotted line shows the prospect of XENONnT [56]. The red dashed line shows the prospect of $W^{\prime} \operatorname{search}[44]$. In the left panel, the green line shows the value of $\Gamma_{W^{\prime}} / m_{W^{\prime}}$, and $\Gamma_{W^{\prime}}>m_{W^{\prime}}$ in the green shaded region. The red shaded region in the right panel is already constrained by the $W^{\prime}$ search [42]. The black shaded regions in both panels are excluded by the perturbative unitarity of the scalar quartic couplings. The blue-solid contours in the right panel show the value of $g_{0}$. 
the dark matter but has almost degenerate mass. In this case, pairs of dark matter particles can annihilate into a heavy triplet and a SM particle. This process is efficient, and the measured value of dark matter energy density is explained for $m_{V} \gtrsim 4 \mathrm{TeV}$. The upper bound on the mass of the dark matter is imposed by the perturbative unitarity bound of the gauge couplings, $m_{V} \lesssim 19 \mathrm{TeV}$. The HL-LHC can test this scenario up to $6 \mathrm{TeV}$. The second scenario is for $m_{W^{\prime}} \simeq 2 m_{V}$ that utilizes the $W^{\prime}$ resonance in the (co)annihilation processes of pairs of dark matter particles. In this case, the gauge couplings are well in the perturbative regime. The third scenario is for $m_{W^{\prime}} \gg m_{V}$. In this scenario, the mass of the dark matter is almost uniquely determined with the assumption that the relic abundance explains the full of the dark matter energy density, $m_{V} \simeq 3 \mathrm{TeV}$. This last scenario is similar to other $\mathrm{SU}(2)_{L}$-triplet dark matter models. The mass of the $W^{\prime}$ is bounded by the condition that $\Gamma_{W^{\prime}}<m_{W^{\prime}}$, and we find $m_{W^{\prime}} \lesssim 15 \mathrm{TeV}$ in the small scalar mixing limit.

Although we do not need to rely on the Higgs portal interactions in this model, it predicts the signal for the direct detection experiments, and thus we also discussed the effects of the scalar mixing. We found that the perturbative unitarity bounds for the scalar quartic couplings give a stronger constraint on the mixing. We also found that the model is testable at the XENONnT experiment if $\left|\phi_{h}\right| \gtrsim 0.03$.

Since our dark matter interacts with the electroweak gauge bosons and is much heavier than them, the Sommerfeld enhancement is expected to give significant effects [57-61]. It may alter our results for the relic abundance. The effect is also important to test this model by the indirect detection experiments. We leave this to further study.

\section{Acknowledgments}

This work was supported by JSPS KAKENHI Grant Number 16K17715 and 19H04615 [T.A.], and by Grant-in-Aid for Scientific research from the Ministry of Education, Science, Sports, and Culture (MEXT), Japan, No. 16H06492 [J.H.]. The work is also supported by World Premier International Research Center Initiative (WPI Initiative), MEXT, Japan [J.H.] and by JSPS Core-to-Core Program (grant number:JPJSCCA20200002).

\section{A Some details in the gauge sectors}

The mass eigenstates are given by

$$
\begin{gathered}
\left(\begin{array}{c}
V_{\mu}^{ \pm} \\
W_{\mu}^{ \pm} \\
W_{\mu}^{ \pm}
\end{array}\right)=\left(\begin{array}{ccc}
\omega_{V}^{0} & \omega_{V}^{1} & \omega_{V}^{2} \\
\omega_{W}^{0} & \omega_{W}^{1} & \omega_{W}^{2} \\
\omega_{W^{\prime}}^{0} & \omega_{W^{\prime}}^{1} & \omega_{W^{\prime}}^{2}
\end{array}\right)\left(\begin{array}{l}
W_{0 \mu}^{ \pm} \\
W_{1 \mu}^{ \pm} \\
W_{2 \mu}^{\mu}
\end{array}\right)=\left(\begin{array}{cccc}
\frac{1}{\sqrt{2}} & 0 & -\frac{1}{\sqrt{2}} \\
\frac{\sin \phi_{ \pm}}{\sqrt{2}} & \cos \phi_{ \pm} & \frac{\sin \phi_{ \pm}}{\sqrt{2}} \\
\frac{\cos \phi_{ \pm}}{\sqrt{2}} & -\sin \phi_{ \pm} & \frac{\cos \phi_{ \pm}}{\sqrt{2}}
\end{array}\right)\left(\begin{array}{c}
W_{0 \mu}^{ \pm} \\
W_{1 \mu}^{ \pm} \\
W_{2 \mu}^{\mu}
\end{array}\right), \\
\left(\begin{array}{c}
V_{\mu}^{0} \\
A_{\mu} \\
Z_{\mu} \\
Z_{\mu}^{\prime \pm}
\end{array}\right)=\left(\begin{array}{cccc}
\omega_{V}^{0} & \omega_{V}^{1} & \omega_{V}^{2} & \omega_{V}^{B} \\
\omega_{\gamma}^{0} & \omega_{\gamma}^{1} & \omega_{\gamma}^{2} & \omega_{\gamma}^{B} \\
\omega_{Z}^{0} & \omega_{Z}^{1} & \omega_{Z}^{2} & \omega_{Z}^{B} \\
\omega_{Z^{\prime}}^{0} & \omega_{Z^{\prime}}^{1} & \omega_{Z^{\prime}}^{2} & \omega_{Z^{\prime}}^{B}
\end{array}\right)\left(\begin{array}{c}
W_{0 \mu}^{3} \\
W_{1 \mu}^{3} \\
W_{2 \mu}^{3} \\
B_{\mu}
\end{array}\right)=\left(\begin{array}{cccc}
\frac{1}{\sqrt{2}} & 0 & -\frac{1}{\sqrt{2}} & 0 \\
\frac{e}{g_{0}} & \frac{e}{g_{1}} & \frac{e}{g_{0}} & \frac{e}{g^{\prime}} \\
\omega_{Z}^{0} & \omega_{Z}^{1} & \omega_{Z}^{0} & \omega_{Z}^{B} \\
\omega_{Z^{\prime}}^{0} & \omega_{Z^{\prime}}^{1} & \omega_{Z^{\prime}}^{0} & \omega_{Z^{\prime}}^{B}
\end{array}\right)\left(\begin{array}{c}
W_{0 \mu}^{3} \\
W_{1 \mu}^{3} \\
W_{2 \mu}^{3} \\
B_{\mu}
\end{array}\right),
\end{gathered}
$$


where

$$
\begin{aligned}
e & =\left(\frac{2}{g_{0}^{2}}+\frac{1}{g_{1}^{2}}+\frac{1}{g^{\prime 2}}\right)^{-1 / 2}, \\
\omega_{Z}^{0}=\omega_{Z}^{2} & =\frac{e g_{1}}{\sqrt{g_{0}^{2}+2 g_{1}^{2} g^{\prime}}} \cos \phi_{0}+\frac{g_{0}}{\sqrt{2\left(g_{0}^{2}+2 g_{1}^{2}\right)}} \sin \phi_{0}, \\
\omega_{Z}^{1} & =\frac{e g_{0}}{\sqrt{g_{0}^{2}+2 g_{1}^{2} g^{\prime}}} \cos \phi_{0}-\frac{\sqrt{2} g_{1}}{\sqrt{g_{0}^{2}+2 g_{1}^{2}}} \sin \phi_{0}, \\
\omega_{Z}^{B} & =-\frac{e \sqrt{g_{0}^{2}+2 g_{1}^{2}}}{g_{0} g_{1}} \cos \phi_{0}, \\
\omega_{Z^{\prime}}^{0}=\omega_{Z^{\prime}}^{2} & =\frac{g_{0}}{\sqrt{2\left(g_{0}^{2}+2 g_{1}^{2}\right)}} \cos \phi_{0}-\frac{e g_{1}}{\sqrt{g_{0}^{2}+2 g_{1}^{2}} g^{\prime}} \sin \phi_{0}, \\
\omega_{Z^{\prime}}^{1} & =-\frac{\sqrt{2} g_{1}}{\sqrt{g_{0}^{2}+2 g_{1}^{2}}} \cos \phi_{0}-\frac{e g_{0}}{\sqrt{g_{0}^{2}+2 g_{1}^{2}} g^{\prime}} \sin \phi_{0}, \\
\omega_{Z^{\prime}}^{B} & =\frac{e \sqrt{g_{0}^{2}+2 g_{1}^{2}}}{g_{0} g_{1}} \sin \phi_{0} .
\end{aligned}
$$

Here we introduce $\phi_{ \pm}$and $\phi_{0}$ that satisfy

$$
\begin{aligned}
& \frac{1}{4}\left(\begin{array}{cc}
g_{1}^{2}\left(v^{2}+2 v_{\Phi}^{2}\right) & -\sqrt{2} g_{0} g_{1} v_{\Phi}^{2} \\
-\sqrt{2} g_{0} g_{1} v_{\Phi}^{2} & g_{0}^{2} v_{\Phi}^{2}
\end{array}\right)\left(\begin{array}{cc}
\cos \phi_{ \pm} & -\sin \phi_{ \pm} \\
\sin \phi_{ \pm} & \cos \phi_{ \pm}
\end{array}\right) \\
& =\left(\begin{array}{cc}
\cos \phi_{ \pm} & -\sin \phi_{ \pm} \\
\sin \phi_{ \pm} & \cos \phi_{ \pm}
\end{array}\right)\left(\begin{array}{cc}
m_{W}^{2} & 0 \\
0 & m_{W^{\prime}}^{2}
\end{array}\right), \\
& \frac{1}{4}\left(\begin{array}{cc}
\frac{g_{0}^{2} g_{1}^{2} g^{\prime 2}}{e^{2}\left(g_{0}^{2}+2 g_{1}^{2}\right)} v^{2} & -\frac{\sqrt{2} g_{0} g_{1}^{3} g^{\prime}}{e\left(g_{0}^{2}+2 g_{1}^{2}\right)} v^{2} \\
-\frac{\sqrt{2} g g_{1}^{3} g^{\prime}}{e\left(g_{0}^{2}+2 g_{1}^{2}\right)} v^{2} & \frac{\left(g_{0}^{2}+2 g_{1}^{2}\right)^{2} v_{\Phi}^{2}+2 g_{1}^{4} v^{2}}{\left(g_{0}^{2}+2 g_{1}^{2}\right)}
\end{array}\right)\left(\begin{array}{cc}
\cos \phi_{0} & -\sin \phi_{0} \\
\sin \phi_{0} & \cos \phi_{0}
\end{array}\right) \\
& =\left(\begin{array}{cc}
\cos \phi_{0} & -\sin \phi_{0} \\
\sin \phi_{0} & \cos \phi_{0}
\end{array}\right)\left(\begin{array}{cc}
m_{Z}^{2} & 0 \\
0 & m_{Z^{\prime}}^{2}
\end{array}\right) .
\end{aligned}
$$

We find

$$
\cos ^{2} \phi_{ \pm}=\frac{m_{V^{ \pm}}^{2}-m_{W}^{2}}{m_{W^{\prime}}^{2}-m_{W}^{2}} .
$$

One can always choose $\cos \phi_{ \pm}>0$ as a convention, and thus

$$
\cos \phi_{ \pm}=\sqrt{\frac{m_{V^{ \pm}}^{2}-m_{W}^{2}}{m_{W^{\prime}}^{2}-m_{W}^{2}}}, \quad \sin \phi_{ \pm}=\sqrt{\frac{m_{W^{\prime}}^{2}-m_{V^{ \pm}}^{2}}{m_{W^{\prime}}^{2}-m_{W}^{2}}} .
$$

We also find

$$
\begin{aligned}
& m_{W}^{2}=\frac{1}{8}\left\{g_{1}^{2} v^{2}+\left(g_{0}^{2}+2 g_{1}^{2}\right) v_{\Phi}^{2}-\sqrt{-4 g_{0}^{2} g_{1}^{2} v^{2} v_{\Phi}^{2}+\left[g_{1}^{2} v^{2}+\left(g_{0}^{2}+2 g_{1}^{2}\right) v_{\Phi}^{2}\right]^{2}}\right\} \\
& m_{W^{\prime}}^{2}=\frac{1}{8}\left\{g_{1}^{2} v^{2}+\left(g_{0}^{2}+2 g_{1}^{2}\right) v_{\Phi}^{2}+\sqrt{-4 g_{0}^{2} g_{1}^{2} v^{2} v_{\Phi}^{2}+\left[g_{1}^{2} v^{2}+\left(g_{0}^{2}+2 g_{1}^{2}\right) v_{\Phi}^{2}\right]^{2}}\right\}
\end{aligned}
$$


For $v_{\Phi} \gg v$, the mixing angles are given by

$$
\begin{aligned}
\cos \phi_{ \pm} & =\frac{g_{0}}{\sqrt{g_{0}^{2}+2 g_{1}^{2}}}+\mathcal{O}\left(v_{\Phi}^{-2}\right) \simeq \frac{m_{V}}{m_{Z^{\prime}}} \\
\sin \phi_{ \pm} & =\frac{\sqrt{2} g_{1}}{\sqrt{g_{0}^{2}+2 g_{1}^{2}}}+\mathcal{O}\left(v_{\Phi}^{-2}\right) \simeq \sqrt{1-\frac{m_{V}^{2}}{m_{Z^{\prime}}^{2}}} \\
\cos \phi_{0} & =1+\mathcal{O}\left(v_{\Phi}^{-4}\right), \\
\sin \phi_{0} & =\frac{\sqrt{2} g_{0} g_{1}^{3} g^{\prime}}{e\left(g_{0}^{2}+2 g_{1}^{2}\right)^{2}} \frac{v^{2}}{v_{\Phi}^{2}}+\mathcal{O}\left(v_{\Phi}^{-4}\right) .
\end{aligned}
$$

\section{B Would-be NG bosons}

The mass matrices for the gauge bosons are given by

$$
\mathcal{M}_{C}^{2}=Q_{W}^{t} Q_{W}, \quad \mathcal{M}_{N}^{2}=Q_{Z}^{t} Q_{Z},
$$

where

$$
\begin{aligned}
Q_{W} & =\frac{1}{2}\left(\begin{array}{ccc}
g_{0} v_{\Phi} & -g_{1} v_{\Phi} & 0 \\
0 & g_{1} v & 0 \\
0 & -g_{1} v_{\Phi} & g_{0} v_{\Phi}
\end{array}\right), \\
Q_{Z} & =\frac{1}{2}\left(\begin{array}{cccc}
g_{0} v_{\Phi} & -g_{1} v_{\Phi} & 0 & 0 \\
0 & g_{1} v & 0 & -g^{\prime} v \\
0 & -g_{1} v_{\Phi} & g_{0} v_{\Phi} & 0
\end{array}\right) .
\end{aligned}
$$

In the $R_{\xi}$ gauge, the mass terms are given by

$$
-\left(\begin{array}{lll}
\pi_{1}^{+} & \pi_{3}^{+} & \pi_{2}^{+}
\end{array}\right) \xi Q_{W} Q_{W}^{t}\left(\begin{array}{c}
\pi_{1}^{-} \\
\pi_{3}^{+} \\
\pi_{2}^{-}
\end{array}\right)-\frac{1}{2}\left(\begin{array}{lll}
\pi_{1}^{0} & \pi_{3}^{0} & \pi_{2}^{0}
\end{array}\right) \xi Q_{Z} Q_{Z}^{t}\left(\begin{array}{c}
\pi_{1}^{0} \\
\pi_{3}^{0} \\
\pi_{2}^{0}
\end{array}\right) .
$$

The eigenvectors of the mass matrices are

$$
\begin{aligned}
Q_{W}^{t} Q_{W} \vec{\omega}_{X} & =m_{X}^{2} \vec{\omega}_{X}, \\
Q_{W} Q_{W}^{t} \vec{\omega}_{\pi_{X}} & =m_{X}^{2} \vec{\omega}_{\pi_{X}}, \\
Q_{Z}^{t} Q_{Z} \vec{\omega}_{X} & =m_{X}^{2} \vec{\omega}_{X}, \\
Q_{Z} Q_{Z}^{t} \vec{\omega}_{\pi_{X}} & =m_{X}^{2} \vec{\omega}_{\pi_{X}} .
\end{aligned}
$$

Multiplying $Q_{W}$ to eq. (B.5) and comparing it with eq. (B.6), one can find that $Q_{W} \vec{\omega}_{X} \propto$ $\vec{\omega}_{\pi_{X}}$. Note that $\left(Q_{W} \vec{\omega}_{X}\right)^{t}\left(Q_{W} \vec{\omega}_{X}\right)=\vec{\omega}_{X}^{t} Q_{W}^{t} Q_{W} \vec{\omega}_{X}=m_{X}^{2}$ and $\vec{\omega}_{\pi_{X}}^{t} \vec{\omega}_{\pi_{X}}=1$. Similar relations are also found in the neutral sector. Finally, we find

$$
\begin{aligned}
Q_{W} \vec{\omega}_{X} & =m_{X} \vec{\omega}_{\pi_{X}}, \\
Q_{Z} \vec{\omega}_{X} & =m_{X} \vec{\omega}_{\pi_{X}} .
\end{aligned}
$$


These relations are useful to obtain the Fermi constant and some relation among couplings. For example, we use

$$
g_{1} v \omega_{X}^{1}=2 m_{X} \omega_{\pi_{X}}^{3}
$$

to obtain the Fermi constant.

The mixing angles for the charged NG-bosons are given by

$$
\left(\begin{array}{lll}
\vec{\omega}_{\pi_{V^{ \pm}}} & \vec{\omega}_{\pi_{W}} & \vec{\omega}_{\pi_{W^{\prime}}}
\end{array}\right)=\left(\begin{array}{lll}
\omega_{\pi_{V^{ \pm}}}^{1} & \omega_{\pi_{W}}^{1} & \omega_{\pi_{W^{\prime}}}^{1} \\
\omega_{\pi_{V^{ \pm}}}^{3} & \omega_{\pi_{W}}^{3} & \omega_{\pi_{W^{\prime}}}^{3} \\
\omega_{\pi_{V^{ \pm}}}^{2} & \omega_{\pi_{W}}^{2} & \omega_{\pi_{W^{\prime}}}^{2}
\end{array}\right)=\left(\begin{array}{ccc}
\frac{1}{\sqrt{2}} & \frac{\sin \phi_{\pi}}{\sqrt{2}} & \frac{\cos \phi_{\pi}}{\sqrt{2}} \\
0 & \cos \phi_{\pi} & -\sin \phi_{\pi} \\
-\frac{1}{\sqrt{2}} & \frac{\sin \phi_{\pi}}{\sqrt{2}} & \frac{\cos \phi_{\pi}}{\sqrt{2}}
\end{array}\right)
$$

where $\sin \phi_{\pi}$ and $\cos \phi_{\pi}$ satisfy

$$
\frac{1}{4}\left(\begin{array}{cc}
g_{1}^{2} v^{2} & -\sqrt{2} g_{1}^{2} v v_{\Phi} \\
-\sqrt{2} g_{1}^{2} v v_{\Phi} & \left(g_{0}^{2}+2 g_{1}^{2}\right) v_{\Phi}^{2}
\end{array}\right)\left(\begin{array}{cc}
\cos \phi_{\pi} & -\sin \phi_{\pi} \\
\sin \phi_{\pi} & \cos \phi_{\pi}
\end{array}\right)=\left(\begin{array}{cc}
\cos \phi_{\pi} & -\sin \phi_{\pi} \\
\sin \phi_{\pi} & \cos \phi_{\pi}
\end{array}\right)\left(\begin{array}{cc}
m_{W}^{2} & 0 \\
0 & m_{W^{\prime}}^{2}
\end{array}\right) .
$$

Comparing eqs. (B.12) and (B.9), we find

$$
\begin{aligned}
\cos \phi_{\pi} & =\frac{g_{1} v}{2 m_{W}} \cos \phi_{ \pm}, \\
\sin \phi_{\pi} & =\frac{g_{1} v}{2 m_{W^{\prime}}} \sin \phi_{ \pm} .
\end{aligned}
$$

The mixing angles for the neutral NG-bosons are given by

$$
\left(\begin{array}{lll}
\vec{\omega}_{\pi_{V^{0}}} & \vec{\omega}_{\pi_{Z}} & \vec{\omega}_{\pi_{Z^{\prime}}}
\end{array}\right)=\left(\begin{array}{ccc}
\omega_{\pi_{V^{0}}}^{1} & \omega_{\pi_{Z}}^{1} & \omega_{\pi_{Z^{\prime}}}^{1} \\
\omega_{\pi_{V^{0}}}^{3} & \omega_{\pi_{Z}}^{3} & \omega_{\pi_{Z^{\prime}}}^{3} \\
\omega_{\pi_{V^{0}}}^{2} & \omega_{\pi_{Z}}^{2} & \omega_{\pi_{Z^{\prime}}}^{2}
\end{array}\right)=\left(\begin{array}{ccc}
\frac{1}{\sqrt{2}} & \frac{\sin \phi_{\pi_{0}}}{\sqrt{2}} & \frac{\cos \phi_{\pi_{0}}}{\sqrt{2}} \\
0 & \cos \phi_{\pi_{0}} & -\sin \phi_{\pi_{0}} \\
-\frac{1}{\sqrt{2}} & \frac{\sin \phi_{\pi_{0}}}{\sqrt{2}} & \frac{\cos \phi_{\pi_{0}}}{\sqrt{2}}
\end{array}\right),
$$

where $\sin \phi_{\pi_{0}}$ and $\cos \phi_{\pi_{0}}$ satisfy

$$
\frac{1}{4}\left(\begin{array}{cc}
\left(g_{1}^{2}+g^{\prime 2}\right) v^{2} & -\sqrt{2} g_{1}^{2} v v_{\Phi} \\
-\sqrt{2} g_{1}^{2} v v_{\Phi} & \left(g_{0}^{2}+2 g_{1}^{2}\right) v_{\Phi}^{2}
\end{array}\right)\left(\begin{array}{cc}
\cos \phi_{\pi_{0}} & -\sin \phi_{\pi_{0}} \\
\sin \phi_{\pi_{0}} & \cos \phi_{\pi_{0}}
\end{array}\right)=\left(\begin{array}{cc}
\cos \phi_{\pi_{0}} & -\sin \phi_{\pi_{0}} \\
\sin \phi_{\pi_{0}} & \cos \phi_{\pi_{0}}
\end{array}\right)\left(\begin{array}{cc}
m_{Z}^{2} & 0 \\
0 & m_{Z^{\prime}}^{2}
\end{array}\right) .
$$

Comparing eqs. (B.16) and (B.10), we find

$$
\begin{aligned}
\cos \phi_{\pi_{0}} & =\frac{v}{2 m_{Z}}\left(g_{1} \omega_{Z}^{1}-g^{\prime} \omega_{Z}^{B}\right), \\
\sin \phi_{\pi_{0}} & =-\frac{v}{2 m_{Z^{\prime}}}\left(g_{1} \omega_{Z^{\prime}}^{1}-g^{\prime} \omega_{Z^{\prime}}^{B}\right) .
\end{aligned}
$$

\section{Fermi constant}

The Fermi constant is defined by the muon decay, $\mu \rightarrow \nu_{\mu} e \bar{\nu}_{e}$. There is a $W^{\prime}$ exchanging diagram as well as the $W$-exchanging diagram. We have to add both contributions. We 
can simplify the calculation by using the relation between the mixing angles in the gauge sector and NG-boson sector. The Fermi constant is given by

$$
\begin{aligned}
\sqrt{2} G_{F} & \equiv \sum_{X=W, W^{\prime}} \frac{g_{X \overline{\nu_{\mu}}} g_{X \bar{e} \nu_{e}}}{4 m_{X}^{2}} \\
& =\sum_{X=W, V, W^{\prime}} \frac{\left(g_{1} \omega_{X}^{1}\right)^{2}}{4 m_{X}^{2}} \\
& =\sum_{X=W, V, W^{\prime}} \frac{\left(\omega_{\pi_{X}}^{3}\right)^{2}}{v^{2}} \\
& =\frac{1}{v^{2}} .
\end{aligned}
$$

In the last line, we used that $\sum_{X} \omega_{\pi_{X}}^{j} \omega_{\pi_{X}}^{k}=\delta^{j k}$. Therefore, we find

$$
\sqrt{2} G_{F}=\frac{1}{v^{2}} .
$$

Open Access. This article is distributed under the terms of the Creative Commons Attribution License (CC-BY 4.0), which permits any use, distribution and reproduction in any medium, provided the original author(s) and source are credited.

\section{References}

[1] Planck collaboration, Planck 2018 results. VI. Cosmological parameters, arXiv: 1807.06209 [INSPIRE].

[2] B.W. Lee and S. Weinberg, Cosmological lower bound on heavy neutrino masses, Phys. Rev. Lett. 39 (1977) 165 [INSPIRE].

[3] XENON collaboration, Dark matter search results from a one ton-year exposure of XENON1T, Phys. Rev. Lett. 121 (2018) 111302 [arXiv:1805.12562] [INSPIRE].

[4] S. Ipek, D. McKeen and A.E. Nelson, A renormalizable model for the Galactic Center gamma ray excess from dark matter annihilation, Phys. Rev. D 90 (2014) 055021 [arXiv: 1404.3716] [INSPIRE].

[5] K. Ghorbani, Fermionic dark matter with pseudo-scalar Yukawa interaction, JCAP 01 (2015) 015 [arXiv: 1408.4929] [INSPIRE].

[6] S. Baek, P. Ko and J. Li, Minimal renormalizable simplified dark matter model with a pseudoscalar mediator, Phys. Rev. D 95 (2017) 075011 [arXiv:1701.04131] [INSPIRE].

[7] C. Gross, O. Lebedev and T. Toma, Cancellation mechanism for dark-matter-nucleon interaction, Phys. Rev. Lett. 119 (2017) 191801 [arXiv:1708.02253] [INSPIRE].

[8] Y. Abe, T. Toma and K. Tsumura, Pseudo-Nambu-Goldstone dark matter from gauged $\mathrm{U}(1)_{B-L}$ symmetry, JHEP 05 (2020) 057 [arXiv: 2001. 03954] [INSPIRE].

[9] N. Okada, D. Raut and Q. Shafi, Pseudo-Goldstone dark matter in gauged B - L extended standard model, arXiv:2001.05910 [INSPIRE].

[10] A. Ahmed, S. Najjari and C.B. Verhaaren, A minimal model for neutral naturalness and pseudo-Nambu-Goldstone dark matter, JHEP 06 (2020) 007 [arXiv:2003.08947] [INSPIRE]. 
[11] N. Maru, N. Okada and S. Okada, $\mathrm{SU}(2)_{L}$ doublet vector dark matter from gauge-Higgs unification, Phys. Rev. D 98 (2018) 075021 [arXiv: 1803.01274] [InSPIRE].

[12] A. Belyaev et al., Minimal spin-one isotriplet dark matter, Phys. Rev. D 99 (2019) 115003 [arXiv: 1808.10464] [INSPIRE].

[13] G. Servant and T.M.P. Tait, Is the lightest Kaluza-Klein particle a viable dark matter candidate?, Nucl. Phys. B 650 (2003) 391 [hep-ph/0206071] [InSPIRE].

[14] S. Kanemura, S. Matsumoto, T. Nabeshima and N. Okada, Can WIMP dark matter overcome the nightmare scenario?, Phys. Rev. D 82 (2010) 055026 [arXiv:1005.5651] [INSPIRE].

[15] O. Lebedev, H.M. Lee and Y. Mambrini, Vector Higgs-portal dark matter and the invisible Higgs, Phys. Lett. B 707 (2012) 570 [arXiv:1111.4482] [InSPIRE].

[16] T. Abe, M. Kakizaki, S. Matsumoto and O. Seto, Vector WIMP miracle, Phys. Lett. B 713 (2012) 211 [arXiv: 1202.5902] [INSPIRE].

[17] Y. Farzan and A.R. Akbarieh, VDM: a model for Vector Dark Matter, JCAP 10 (2012) 026 [arXiv: 1207.4272] [INSPIRE].

[18] S. Baek, P. Ko, W.-I. Park and E. Senaha, Higgs portal vector dark matter: revisited, JHEP 05 (2013) 036 [arXiv: 1212.2131] [INSPIRE].

[19] J.M. Hyde, A.J. Long and T. Vachaspati, Dark strings and their couplings to the standard model, Phys. Rev. D 89 (2014) 065031 [arXiv:1312.4573] [INSPIRE].

[20] P. Ko, W.-I. Park and Y. Tang, Higgs portal vector dark matter for GeV scale $\gamma$-ray excess from galactic center, JCAP 09 (2014) 013 [arXiv: 1404.5257] [INSPIRE].

[21] S. Baek, P. Ko and W.-I. Park, Invisible Higgs decay width vs. dark matter direct detection cross section in Higgs portal dark matter models, Phys. Rev. D 90 (2014) 055014 [arXiv: 1405.3530] [INSPIRE].

[22] J.-H. Yu, Vector fermion-portal dark matter: direct detection and Galactic Center gamma-ray excess, Phys. Rev. D 90 (2014) 095010 [arXiv:1409.3227] [INSPIRE].

[23] C.-R. Chen, Y.-K. Chu and H.-C. Tsai, An elusive vector dark matter, Phys. Lett. B 741 (2015) 205 [arXiv: 1410.0918] [INSPIRE].

[24] T. Hambye, Hidden vector dark matter, JHEP 01 (2009) 028 [arXiv:0811.0172] [INSPIRE].

[25] H. Zhang, C.S. Li, Q.-H. Cao and Z. Li, A dark matter model with non-Abelian gauge symmetry, Phys. Rev. D 82 (2010) 075003 [arXiv:0910.2831] [InSPIRE].

[26] J. Diaz-Cruz and E. Ma, Neutral SU(2) gauge extension of the standard model and a vector-boson dark-matter candidate, Phys. Lett. B 695 (2011) 264 [arXiv:1007.2631] [INSPIRE].

[27] S. Bhattacharya, J. Diaz-Cruz, E. Ma and D. Wegman, Dark vector-gauge-boson model, Phys. Rev. D 85 (2012) 055008 [arXiv:1107.2093] [INSPIRE].

[28] T. Hambye and A. Strumia, Dynamical generation of the weak and dark matter scale, Phys. Rev. D 88 (2013) 055022 [arXiv: 1306.2329] [INSPIRE].

[29] H. Davoudiasl and I.M. Lewis, Dark matter from hidden forces, Phys. Rev. D 89 (2014) 055026 [arXiv: 1309.6640] [INSPIRE]. 
[30] S. Baek, P. Ko and W.-I. Park, Hidden sector monopole, vector dark matter and dark radiation with Higgs portal, JCAP 10 (2014) 067 [arXiv:1311.1035] [INSPIRE].

[31] V.V. Khoze and G. Ro, Dark matter monopoles, vectors and photons, JHEP 10 (2014) 061 [arXiv:1406.2291] [INSPIRE].

[32] S. Fraser, E. Ma and M. Zakeri, $\mathrm{SU}(2)_{N}$ model of vector dark matter with a leptonic connection, Int. J. Mod. Phys. A 30 (2015) 1550018 [arXiv:1409.1162] [InSPIRE].

[33] A. Karam and K. Tamvakis, Dark matter and neutrino masses from a scale-invariant multi-Higgs portal, Phys. Rev. D 92 (2015) 075010 [arXiv:1508.03031] [InSPIRE].

[34] B. Barman, S. Bhattacharya, S.K. Patra and J. Chakrabortty, Non-Abelian vector boson dark matter, its unified route and signatures at the LHC, JCAP 12 (2017) 021 [arXiv: 1704.04945] [INSPIRE].

[35] B. Barman, S. Bhattacharya and M. Zakeri, Multipartite dark matter in $\mathrm{SU}(2)_{N}$ extension of standard model and signatures at the LHC, JCAP 09 (2018) 023 [arXiv:1806.01129] [INSPIRE].

[36] B. Barman, S. Bhattacharya and M. Zakeri, Non-Abelian vector boson as FIMP dark matter, JCAP 02 (2020) 029 [arXiv: 1905.07236] [INSPIRE].

[37] E. Ma, [SU(2)] ${ }^{3}$ dark matter, Phys. Lett. B 780 (2018) 533 [arXiv:1712.08994] [INSPIRE].

[38] C.T. Hill, S. Pokorski and J. Wang, Gauge invariant effective Lagrangian for Kaluza-Klein modes, Phys. Rev. D 64 (2001) 105005 [hep-th/0104035] [INSPIRE].

[39] N. Arkani-Hamed, A.G. Cohen and H. Georgi, (De)constructing dimensions, Phys. Rev. Lett. 86 (2001) 4757 [hep-th/0104005] [INSPIRE].

[40] H. Georgi, A tool kit for builders of composite models, Nucl. Phys. B 266 (1986) 274 [INSPIRE].

[41] K. Hally, H.E. Logan and T. Pilkington, Constraints on large scalar multiplets from perturbative unitarity, Phys. Rev. D 85 (2012) 095017 [arXiv:1202.5073] [INSPIRE].

[42] ATLAS collaboration, Search for a heavy charged boson in events with a charged lepton and missing transverse momentum from pp collisions at $\sqrt{s}=13 \mathrm{TeV}$ with the ATLAS detector, Phys. Rev. D 100 (2019) 052013 [arXiv:1906.05609] [INSPIRE].

[43] CMS collaboration, Search for high-mass resonances in final states with a lepton and missing transverse momentum at $\sqrt{s}=13 \mathrm{TeV}$, JHEP 06 (2018) 128 [arXiv:1803.11133] [INSPIRE].

[44] ATLAS collaboration, Prospects for searches for heavy $Z^{\prime}$ and $W^{\prime}$ bosons in fermionic final states with the ATLAS experiment at the HL-LHC, Tech. Rep. ATL-PHYS-PUB-2018-044, CERN, Geneva (Dec, 2018).

[45] R. Barbieri, A. Pomarol, R. Rattazzi and A. Strumia, Electroweak symmetry breaking after LEP-1 and LEP-2, Nucl. Phys. B 703 (2004) 127 [hep-ph/0405040] [InSPIRE].

[46] ATLAS collaboration, Combined measurements of Higgs boson production and decay using up to $80 \mathrm{fb}^{-1}$ of proton-proton collision data at $\sqrt{\mathrm{s}}=13 \mathrm{TeV}$ collected with the ATLAS experiment, Phys. Rev. D 101 (2020) 012002 [arXiv:1909.02845] [INSPIRE].

[47] T. Hahn and M. Pérez-Victoria, Automatized one loop calculations in four-dimensions and D-dimensions, Comput. Phys. Commun. 118 (1999) 153 [hep-ph/9807565] [INSPIRE].

[48] M. Cirelli, N. Fornengo and A. Strumia, Minimal dark matter, Nucl. Phys. B 753 (2006) 178 [hep-ph/0512090] [INSPIRE]. 
[49] ATLAS collaboration, Search for long-lived charginos based on a disappearing-track signature in pp collisions at $\sqrt{s}=13 \mathrm{TeV}$ with the ATLAS detector, JHEP 06 (2018) 022 [arXiv: 1712.02118] [INSPIRE].

[50] G. Bélanger et al., MicrOMEGAs5.0: freeze-in, Comput. Phys. Commun. 231 (2018) 173 [arXiv: 1801.03509] [INSPIRE].

[51] J. Hisano, S. Matsumoto, M.M. Nojiri and O. Saito, Direct detection of the Wino and Higgsino-like neutralino dark matters at one-loop level, Phys. Rev. D 71 (2005) 015007 [hep-ph/0407168] [INSPIRE].

[52] J. Hisano, K. Ishiwata and N. Nagata, A complete calculation for direct detection of Wino dark matter, Phys. Lett. B 690 (2010) 311 [arXiv:1004.4090] [INSPIRE].

[53] J. Hisano, K. Ishiwata and N. Nagata, QCD effects on direct detection of Wino dark matter, JHEP 06 (2015) 097 [arXiv: 1504.00915] [INSPIRE].

[54] A. Alloul, N.D. Christensen, C. Degrande, C. Duhr and B. Fuks, FeynRules $2.0-A$ complete toolbox for tree-level phenomenology, Comput. Phys. Commun. 185 (2014) 2250 [arXiv:1310.1921] [INSPIRE].

[55] J. Hisano et al., Non-perturbative effect on thermal relic abundance of dark matter, Phys. Lett. B 646 (2007) 34 [hep-ph/0610249] [INSPIRE].

[56] XENON collaboration, Physics reach of the XENON1T dark matter experiment, JCAP 04 (2016) 027 [arXiv:1512.07501] [INSPIRE].

[57] J. Hisano, S. Matsumoto and M.M. Nojiri, Unitarity and higher order corrections in neutralino dark matter annihilation into two photons, Phys. Rev. D 67 (2003) 075014 [hep-ph/0212022] [INSPIRE].

[58] J. Hisano, S. Matsumoto and M.M. Nojiri, Explosive dark matter annihilation, Phys. Rev. Lett. 92 (2004) 031303 [hep-ph/0307216] [INSPIRE].

[59] J. Hisano, S. Matsumoto, M.M. Nojiri and O. Saito, Non-perturbative effect on dark matter annihilation and gamma ray signature from galactic center, Phys. Rev. D 71 (2005) 063528 [hep-ph/0412403] [INSPIRE].

[60] N. Arkani-Hamed, D.P. Finkbeiner, T.R. Slatyer and N. Weiner, A theory of dark matter, Phys. Rev. D 79 (2009) 015014 [arXiv:0810.0713] [INSPIRE].

[61] K. Blum, R. Sato and T.R. Slatyer, Self-consistent calculation of the sommerfeld enhancement, JCAP 06 (2016) 021 [arXiv: 1603.01383] [INSPIRE]. 